\title{
On spherically symmetric solutions of the Einstein-Euler equations
}

\author{
Tetu Makino
}

\begin{abstract}
We construct spherically symmetric solutions to the Einstein-Euler equations, which give models of gaseous stars in the framework of the general theory of relativity. We assume a realistic barotropic equation of state. Equilibria of the spherically symmetric Einstein-Euler equations are given by the Tolman-OppenheimerVolkoff equations, and time-periodic solutions around the equilibrium of the linearized equations can be considered. Our aim is to find true solutions near these time-periodic approximations. Solutions satisfying a so-called physical boundary condition at the free boundary with the vacuum will be constructed using the Nash-Moser theorem. This work also can be considered as a touchstone in order to estimate the universality of the method which was originally developed for the nonrelativistic Euler-Poisson equations.
\end{abstract}

\section{Introduction}

Recently, U. Brauer and L. Karp [2, Theorem 2.3] established a local existence theorem of solutions to the Cauchy problem for the Einstein-Euler equations, which describes a relativistic self-gravitating perfect fluid having density either compactly supported or falling off at infinity in an appropriate manner. In their work [2] the energy-momentum tensor of the perfect fluid takes the form

$$
T^{\mu \nu}=(\epsilon+P) U^{\mu} U^{\nu}-P g^{\mu \nu},
$$

where $\epsilon=c^{2} \rho$ is the energy density, $P$ is the pressure, and $U^{\mu}$ is the velocity 4 vector. Here it is assumed that $P=K \epsilon^{\gamma}, K>0, \gamma>1$, and the quantity

$$
w:=\epsilon^{\frac{\gamma-1}{2}}=c^{\gamma-1} \rho^{\frac{\gamma-1}{2}}
$$

is introduced. The main result requires that the initial data satisfy $w \in H_{s+1}$ with $s>3 / 2$ so that $w \in C^{1}$ at least.

However, a spherically symmetric equilibrium, which solves the TolmanOppenheimer-Volkoff equation, satisfies $w \sim \operatorname{const}\left(r_{+}-r\right)^{1 / 2}$ as $r \rightarrow r_{+}-0$ provided that the equilibrium has a finite radius $r_{+}$(see Section 3 ). Hence, such an equilibrium is excluded from the class of density distributions admissible to this local existence theorem. We are faced with the same situation in the nonrelativistic problem governed by the Euler-Poisson equations as discussed in [6].

Kyoto Journal of Mathematics, Vol. 56, No. 2 (2016), 243-282

DOI $10.1215 / 21562261-3478880$, (C) 2016 by Kyoto University

Received October 14, 2014. Revised February 6, 2015. Accepted February 13, 2015.

2010 Mathematics Subject Classification: Primary 35L05, 35L52, 35L57, 35L70; Secondary 76L10, $76 \mathrm{~N} 15,83 \mathrm{C} 05,85 \mathrm{~A} 30$. 
Recently, this trouble was partially overcome by [8] in the Euler-Poisson equations for the nonrelativistic case. So, a similar discussion is required for the relativistic problem. That is the aim of this article.

\section{Spherically symmetric evolution equations}

The Einstein equations read ([5, (95.5)])

$$
R_{\mu \nu}-\frac{1}{2} g_{\mu \nu} R=\frac{8 \pi G}{c^{4}} T_{\mu \nu}
$$

Here $R_{\mu \nu}$ is the Ricci tensor, $R$ is the scalar curvature $g^{\alpha \beta} R_{\alpha \beta}$ associated with the metric

$$
d s^{2}=g_{\mu \nu} d x^{\mu} d x^{\nu}
$$

$T^{\mu \nu}$ is the energy-momentum tensor of the matter, $G$ is the constant of gravitation $\left(6.67 \times 10^{-8} \mathrm{~cm}^{3} / \mathrm{g} \cdot \mathrm{s}^{2}\right)$, and $c$ is the speed of light $\left(3.00 \times 10^{10} \mathrm{~cm} / \mathrm{s}\right)$. The Einstein equations (2.1) imply the Euler equations

$$
\nabla_{\nu} T^{\mu \nu}=0
$$

where $\nabla$ denotes the covariant derivative associated with the metric (2.2). The details can be found in [5] or [11].

The energy-momentum tensor of a perfect fluid is given by $([5,(94.4)])$

$$
T^{\mu \nu}=\left(c^{2} \rho+P\right) U^{\mu} U^{\nu}-P g^{\mu \nu},
$$

where $\rho$ is the mass density, $P$ is the pressure, and $U^{\mu}$ stands for the 4-dimensional velocity vector such that $U^{\mu} U_{\mu}=1$. In this article we always assume the following.

\section{ASSUMPTION 1}

We assume that $P$ is a given analytic function of $\rho>0$ such that $0<P, 0<$ $d P / d \rho<c^{2}$ for $\rho>0$, and $P \rightarrow 0$ as $\rho \rightarrow+0$.

If we assume spherical symmetry, then the Einstein-Euler equations are reduced as follows. We consider the metric of the form ([5, p. 304, (1)])

$$
d s^{2}=e^{2 F} c^{2} d t^{2}-e^{2 H} d r^{2}-R^{2}\left(d \theta^{2}+\sin ^{2} \theta d \phi^{2}\right),
$$

where $F, H$, and $R$ are functions of $t, r \geq 0$. (Here $R$ does not mean the scalar curvature $g^{\mu \nu} R_{\mu \nu}$.) Then the nonzero components of the Einstein tensor $G_{\mu}^{\nu}:=R_{\mu}^{\nu}-\frac{1}{2} \delta_{\mu}^{\nu} R$, where $R$ is the scalar curvature, are (see [5, p. 305, (2), (3), $(4),(5)])$

$$
\begin{aligned}
& G_{0}^{0}=e^{-2 H}\left(-\frac{R^{\prime 2}}{R^{2}}-2 \frac{R^{\prime \prime}}{R}+2 \frac{H^{\prime} R^{\prime}}{R}\right)+e^{-2 F}\left(\frac{\dot{R}^{2}}{R^{2}}+2 \frac{\dot{H} \dot{R}}{R}\right)+\frac{1}{R^{2}}, \\
& G_{1}^{1}=e^{-2 F}\left(\frac{\dot{R}^{2}}{R^{2}}+2 \frac{\ddot{R}}{R}-2 \frac{\dot{F} \dot{R}}{R}\right)-e^{-2 H}\left(\frac{R^{\prime 2}}{R^{2}}+2 \frac{F^{\prime} R^{\prime}}{R}\right)+\frac{1}{R^{2}}
\end{aligned}
$$




$$
\begin{aligned}
G_{2}^{2}= & G_{3}^{3} \\
= & e^{-2 H}\left(-\frac{R^{\prime \prime}}{R}-F^{\prime \prime}-F^{2}+H^{\prime} F^{\prime}+\frac{H^{\prime} R^{\prime}}{R}-\frac{F^{\prime} R^{\prime}}{R}\right) \\
& +e^{-2 F}\left(\frac{\ddot{R}}{R}+\ddot{H}+\dot{H}^{2}-\dot{H} \dot{F}+\frac{\dot{H} \dot{R}}{R}-\frac{\dot{F} \dot{R}}{R}\right), \\
e^{2 H} G_{0}^{1}= & -e^{2 F} G_{1}^{0}=2\left(\frac{\dot{R}^{\prime}}{R}-\frac{\dot{H} R^{\prime}}{R}-\frac{F^{\prime} \dot{R}}{R}\right) .
\end{aligned}
$$

Here $\dot{A}$ stands for $\partial A / c \partial t$ and $A^{\prime}$ stands for $\partial A / \partial r$. Of course the coordinates $x^{\mu}$ are taken as

$$
x^{0}=c t, \quad x^{1}=r, \quad x^{2}=\theta, \quad x^{3}=\phi .
$$

By the freedom of choice of $r$ we take it in such a way that the flow is apparently static, say, we suppose

$$
U^{0}=e^{-F}, \quad U^{1}=U^{2}=U^{3}=0 .
$$

Then the energy-momentum tensor turns out to be

$$
T_{0}^{0}=c^{2} \rho, \quad T_{1}^{1}=T_{2}^{2}=T_{3}^{3}=-P, \quad T_{0}^{1}=T_{1}^{0}=0 .
$$

The equation $\nabla_{\mu} T_{0}^{\mu}=0$ gives

$$
c^{2} \dot{\rho}+\left(\dot{H}+\frac{2 \dot{R}}{R}\right)\left(c^{2} \rho+P\right)=0,
$$

and the equation $\nabla_{\mu} T_{1}^{\mu}=0$ gives

$$
P^{\prime}+F^{\prime}\left(c^{2} \rho+P\right)=0 .
$$

By integrating (2.9) we can suppose that $F$ is a function of $\rho$ given by

$$
F=F(\rho)=-\int^{\rho} \frac{1}{c^{2} \rho+P} \frac{d P}{d \rho} d \rho .
$$

Let us introduce the variable $m$ by

$$
m=4 \pi \int_{0}^{R} \rho R^{2} d R=4 \pi \int_{0}^{r} \rho R^{2} R^{\prime} d r .
$$

The variable $V$ is defined by

$$
V=c e^{-F} \dot{R}
$$

Then the equation $G_{0}^{1}=0$ turns out to be

$$
\dot{H}=\frac{1}{c} e^{F} \frac{V^{\prime}}{R^{\prime}} .
$$

Substituting (2.12) and (2.13) into (2.8), we have

$$
c^{2} \dot{\rho}=-\frac{1}{c} e^{F}\left(c^{2} \rho+P\right)\left(\frac{V^{\prime}}{R^{\prime}}+\frac{2 V}{R}\right) .
$$

Eliminating the time derivatives from the equation $G_{0}^{0}=\frac{8 \pi G}{c^{2}} \rho$, we have

$$
\frac{8 \pi G}{c^{2}} \rho R^{2} R^{\prime}=\left(-R R^{\prime 2} e^{-2 H}+\frac{1}{c^{2}} R V^{2}+R\right)^{\prime} .
$$


Integrating this, keeping in mind that $R$ should vanish at $r=0$, we get

$$
m=\frac{c^{2} R}{2 G}\left(\frac{V^{2}}{c^{2}}+1-R^{\prime 2} e^{-2 H}\right)
$$

from which we get

$$
e^{2 H}=\left(1+\frac{V^{2}}{c^{2}}-\frac{2 G m}{c^{2} R}\right)^{-1} R^{\prime 2}
$$

Differentiating (2.12) with respect to $t$ and using the equation $G_{1}^{1}=-\frac{8 \pi G}{c^{4}} P$ and (2.15), we obtain

$$
\frac{\dot{V}}{c} e^{-F}=-\frac{G R}{c^{2}}\left(\frac{m}{R^{3}}+\frac{4 \pi P}{c^{2}}\right)-e^{-2 H} \frac{R^{\prime} P^{\prime}}{c^{2} \rho+P},
$$

or from (2.16),

$$
e^{-F} c \dot{V}=-G R\left(\frac{m}{R^{3}}+\frac{4 \pi P}{c^{2}}\right)-\left(1+\frac{V^{2}}{c^{2}}-\frac{2 G m}{c^{2} R}\right) \frac{P^{\prime}}{R^{\prime}\left(\rho+P / c^{2}\right)} .
$$

Differentiating (2.15) with respect to $t$ and using the equation $G_{1}^{0}=0$, we have

$$
\dot{m} e^{-F}=-\frac{4 \pi R^{2}}{c^{3}} P V .
$$

Now (2.12), (2.14), (2.18), and (2.19) govern the evolution of unknowns $R$, $H, \rho, V, m$. The system of equations to be studied is

(2.20a) $e^{-F} c \dot{R}=V$,

(2.20b) $e^{-F} c \dot{\rho}=-\left(\rho+P / c^{2}\right)\left(\frac{V^{\prime}}{R^{\prime}}+\frac{2 V}{R}\right)$,

(2.20c) $e^{-F} c \dot{V}=-G R\left(\frac{m}{R^{3}}+\frac{4 \pi P}{c^{2}}\right)-\left(1+\frac{V^{2}}{c^{2}}-\frac{2 G m}{c^{2} R}\right) \frac{P^{\prime}}{R^{\prime}\left(\rho+P / c^{2}\right)}$,

(2.20d) $e^{-F} c \dot{m}=-\frac{4 \pi}{c^{2}} R^{2} P V$.

Of course, we assume (2.10) and (2.11). The above equations were derived by [10]. The equations (2.20a), (2.20b), (2.20c), and (2.20d) are none other than [10, $(1.12-R),(8.11),(1.12-U),(1.12-m)]$, respectively.

The system of coordinates $(t, r)$ is a comoving Lagrangian system of coordinates moving at each point with the fluid. Therefore, if $\rho>0$ for $0 \leq r<r_{+}$and $\rho=0$ for $r_{+} \leq r$ at $t=0$, then it remains so for all small $t>0$ along the time evolution as long as the $C^{1}$-solution exists, while the surface radius $r_{+}$is constant. (Of course, the value of $R$ at the surface can change in time.) Especially, we have that $m=m_{+}$is constant at $r=r_{+}$for all $t>0$. Hence, we can take $(t, m)$ as another system of comoving Lagrangian coordinates. Then we have the formula

$$
\begin{aligned}
\left(\frac{\partial}{\partial t}\right)_{r} & =\left(\frac{\partial}{\partial t}\right)_{m}-\frac{4 \pi}{c^{2}} e^{F} R^{2} P V \frac{\partial}{\partial m}, \\
\frac{\partial}{\partial r} & =4 \pi \rho R^{2} R^{\prime} \frac{\partial}{\partial m} .
\end{aligned}
$$


Here $(\partial / \partial t)_{r}$ stands for the partial derivative with respect to $t$ keeping $r$ constant, and $(\partial / \partial t)_{m}$ stands for that keeping $m$ constant.

Note that

$$
\frac{\partial R}{\partial m}=\frac{1}{4 \pi \rho R^{2}}
$$

and

$$
\rho=\left(4 \pi R^{2} \frac{\partial R}{\partial m}\right)^{-1}
$$

Thus, (2.20a) reads

$$
e^{-F}\left(\frac{\partial R}{\partial t}\right)_{m}=\left(1+\frac{P}{c^{2} \rho}\right) V
$$

and $(2.20 \mathrm{~d})$ reads

$$
\begin{aligned}
e^{-F}\left(\frac{\partial V}{\partial t}\right)_{m}= & \frac{4 \pi}{c^{2}} R^{2} P V \frac{\partial V}{\partial m}-G R\left(\frac{m}{R^{3}}+\frac{4 \pi P}{c^{2}}\right) \\
& -\left(1+\frac{V^{2}}{c^{2}}-\frac{2 G m}{c^{2} R}\right)\left(1+\frac{P}{c^{2} \rho}\right)^{-1} \cdot 4 \pi R^{2} \frac{\partial P}{\partial m},
\end{aligned}
$$

where we have used the relation

$$
\frac{P^{\prime}}{R^{\prime}}=4 \pi \rho R^{2} \frac{\partial P}{\partial m}
$$

which comes from (2.22).

In summary, the system of equations (2.25)-(2.26) should be solved, while $\rho, P=P(\rho)$ are given functions of $R^{2} \partial R / \partial m$ through (2.24). Moreover, under Assumption 2 specified in the next section, we can put

$$
F=-\frac{u}{c^{2}}+F(0)
$$

in order to fix the idea, where $F(0)$ is a constant and

$$
u=\int_{0}^{\rho} \frac{1}{\rho+P(\rho) / c^{2}} \frac{d P}{d \rho} d \rho
$$

is a given function of $R^{2} \partial R / \partial m$, too (see (2.10)). Hence, the unknown functions are only $(t, m) \mapsto R$ and $(t, m) \mapsto V$.

The system of equations $(2.25)-(2.26)$ will be called $\left(E_{c}\right)$ :

$$
\begin{aligned}
e^{-F} \frac{\partial R}{\partial t}= & \left(1+\frac{P}{c^{2} \rho}\right) V \\
e^{-F} \frac{\partial V}{\partial t}= & \frac{4 \pi}{c^{2}} R^{2} P V \frac{\partial V}{\partial m}-G R\left(\frac{m}{R^{3}}+\frac{4 \pi P}{c^{2}}\right) \\
& -\left(1+\frac{V^{2}}{c^{2}}-\frac{2 G m}{c^{2} R}\right)\left(1+\frac{P}{c^{2} \rho}\right)^{-1} \cdot 4 \pi R^{2} \frac{\partial P}{\partial m}
\end{aligned}
$$

Here we have written $\frac{\partial R}{\partial t}, \frac{\partial V}{\partial t}$ simply instead of $\left(\frac{\partial R}{\partial t}\right)_{m},\left(\frac{\partial V}{\partial t}\right)_{m}$. The nonrelativistic limit as $c \rightarrow+\infty$ is of course $\left(E_{\infty}\right)$ : 


$$
\begin{aligned}
& \frac{\partial R}{\partial t}=V, \\
& \frac{\partial V}{\partial t}=-\frac{G m}{R^{2}}-4 \pi R^{2} \frac{\partial P}{\partial m},
\end{aligned}
$$

which is reduced to the second-order single equation [8, (4)], where $g_{0}, r$ stand for $G, R$, respectively.

\section{SUPPLEMENTARY REMARK 1}

The function $F=F(t, r)$ in the components of the metric (2.5) should satisfy (2.9). Therefore, generally speaking, (2.27) should read

$$
F=-\frac{u}{c^{2}}+F_{+}(t)
$$

where $F_{+}(t)$ is an arbitrary smooth function of $t$, being constant with respect to $r$, or

$$
e^{2 F}=C(t)^{2} \kappa e^{-2 u / c^{2}},
$$

where $\kappa$ is a positive constant which will be specified in the next section (see (3.9)) and where $C(t)$ is an arbitrary positive smooth function of $t$. Then the left-hand sides of (2.25)-(2.26) or $\left(E_{c}\right)$ should be interpreted with

$$
e^{-F} \frac{\partial}{\partial t}=\frac{1}{C(t)} \frac{1}{\sqrt{\kappa}} e^{u / c^{2}} \frac{\partial}{\partial t}
$$

Of course, we can and shall assume that $C(t) \equiv 1$ by taking

$$
t^{*}=t^{*}(t):=\int_{0}^{t} C\left(t^{\prime}\right) d t^{\prime}
$$

instead of $t$, that is, we specify

$$
e^{F}=\sqrt{\kappa} \exp \left(-\frac{u}{c^{2}}\right)
$$

without loss of generality.

In this sense, if we are allowed to forestall the discussion, then we should say that, in order to fix the idea, the definitions of $J, H_{1}, H_{2}$ in Section 6 (see (6.8a), (6.8b), and (6.9)) should be done by using (2.28), where $u$ is a given function of $\rho$ given by

$$
\rho=\bar{\rho}(1+y)^{-2}\left(1+y+r \frac{\partial y}{\partial r}\right)^{-1}
$$

\section{Equilibrium configurations}

Let us consider a solution of (2.20a) $-(2.20 \mathrm{~d})$ which is independent of $t$, that is, $F=F(\rho(r)), H=H(r), \rho=\rho(r), P=P(\rho(r)), V \equiv 0, R \equiv r$. Then the system of equations (2.20a) $-(2.20 \mathrm{~d})$ is reduced to

$$
0=G r\left(\frac{m}{r^{3}}+\frac{4 \pi P}{c^{2}}\right)+\left(1-\frac{2 G m}{c^{2} r}\right) \frac{P^{\prime}}{\rho+P / c^{2}} .
$$


Therefore, the equation to be studied is

$$
\begin{aligned}
& \frac{d m}{d r}=4 \pi r^{2} \rho, \\
& \frac{d P}{d r}=-\left(\rho+P / c^{2}\right) \frac{G\left(m+4 \pi r^{3} P / c^{2}\right)}{r^{2}\left(1-2 G m / c^{2} r\right)} .
\end{aligned}
$$

This equation was first derived by Oppenheimer and Volkoff [12] in 1939.

Let us observe solutions of the Tolman-Oppenheimer-Volkoff equation (3.1). We assume Assumption 1.

\section{PROPOSITION 1}

Let $\rho_{c}(>0)$ and $P_{c}=P\left(\rho_{c}\right)$ be the given central density and central pressure, respectively. Then there is a unique local solution $(m(r), P(r)), 0 \leq r \leq \delta$, of (3.1), $\delta$ being a small positive number, such that $m=0, P=P_{c}$ at $r=0$. Moreover, we have

$$
\begin{aligned}
& m=\frac{4 \pi}{3} \rho_{c} r^{3}+O\left(r^{5}\right), \\
& P=P_{c}-\left(\rho_{c}+P_{c} / c^{2}\right) G\left(4 \pi \rho_{c} / 3+4 \pi P_{c} / c^{2}\right) \frac{r^{2}}{2}+O\left(r^{4}\right)
\end{aligned}
$$

as $r \rightarrow 0$.

A proof can be found in [7].

We consider the domain of (3.1) as $\mathcal{D}:=\{(r, m, P) \mid 0<r<+\infty, 0<P<$ $\left.+\infty, 0<2 G m / c^{2} r<1\right\}$. Prolonging the local solution as long as possible in the domain $\mathcal{D}$, we have $\left(0, r_{+}\right)$for the maximal interval of existence. Here $r_{+} \leq+\infty$ is a constant.

\section{DEFINITION 1}

If $r_{+}=+\infty$, then the solution will be called a long equilibrium with central density $\rho_{c}$. If $r_{+}<+\infty$, then the solution will be called a short equilibrium.

\section{REMARK}

It will be shown that if $r_{+}<+\infty$, then $\rho$ and $P$ tend to 0 but $2 G m / c^{2} r$ tends to a positive number strictly less than 1 as $r \rightarrow r_{+}-0$. In this sense the solution can be said to be "short" if $r_{+}<+\infty$.

The equation of state for neutron stars is given by

$$
\begin{aligned}
P & =K c^{5} \int_{0}^{\zeta} \frac{q^{4} d q}{\left(1+q^{2}\right)^{1 / 2}} \\
& =\frac{3}{8} K c^{5}\left(5\left(1+\zeta^{2}\right)\left(\frac{2}{3} \zeta^{2}-1\right)+\log \left(\zeta+\left(\zeta^{2}+1\right)^{1 / 2}\right)\right)
\end{aligned}
$$




$$
\begin{aligned}
\rho & =3 K c^{3} \int_{0}^{\zeta}\left(1+q^{2}\right)^{1 / 2} q^{2} d q \\
& =\frac{3}{8} K c^{3}\left(2 \zeta^{2}+1\right)\left(\zeta\left(\zeta^{2}+1\right)^{1 / 2}-\log \left(\zeta+\left(\zeta^{2}+1\right)^{1 / 2}\right)\right)
\end{aligned}
$$

See $[15$, p. $188,(6.8 .4),(6.8 .5)]$. In this case we have

$$
P=\frac{1}{5} K^{-2 / 3} \rho^{5 / 3}\left(1+\left[K^{-2 / 3} \rho^{2 / 3} / c^{2}\right]_{1}\right),
$$

where $[X]_{1}$ stands for a convergent power series of the form $\sum_{j \geq 1} a_{j} X^{j}$. Keeping in mind this case, we suppose the following assumption of the behavior of $P(\rho)$ as $\rho \rightarrow 0$.

\section{ASSUMPTION 2}

There are positive constants $A, \gamma$ such that

$$
P=A \rho^{\gamma}\left(1+\left[\rho^{\gamma-1}\right]_{1}\right)
$$

as $\rho \rightarrow+0$, and $1<\gamma<2$.

Under Assumptions 1 and 2 we can introduce the new variable $u$ by

$$
u=\int_{0}^{P} \frac{d P}{\rho+P / c^{2}},
$$

which satisfies

$$
u=\frac{A \gamma}{\gamma-1} \rho^{\gamma-1}\left(1+\left[\rho^{\gamma-1}\right]_{1}\right)
$$

as $\rho \rightarrow+0$. Let $(m(r), P(r)), 0<r<r_{+}$, be an equilibrium, where $\left(0, r_{+}\right)$is the maximal interval of existence. Then the corresponding $u=u(r)$ satisfies

$$
r \frac{d u}{d r}=-\frac{G\left(m+4 \pi r^{3} P / c^{2}\right)}{r\left(1-2 G m / c^{2} r\right)} .
$$

Then $u(r)$ is monotone decreasing, and, moreover, we have the following result.

\section{PROPOSITION 2}

We have $u(r) \rightarrow 0$ as $r \rightarrow r_{+}-0$.

The proof is the same as that of [7, Lemma]. (We do not use the assumption $\gamma>4 / 3$.)

Let us introduce the variables

$$
x=\frac{m}{u r}, \quad y=4 \pi r^{2} \frac{\rho^{2}}{P} .
$$

The equations read

$$
r \frac{d x}{d r}=\alpha(u)-x+x^{2} \tilde{G},
$$




$$
\begin{aligned}
& r \frac{d y}{d r}=y(2-\beta(u) x \tilde{G}), \\
& r \frac{d u}{d r}=-u x \tilde{G},
\end{aligned}
$$

where

$$
\begin{aligned}
& \alpha=\frac{P}{u \rho}=\frac{\gamma-1}{\gamma}+[u]_{1}, \\
& \beta=\left(2 \frac{d P}{d \rho}-\frac{u}{P}\right)=\frac{2-\gamma}{\gamma-1}+[u]_{1}, \\
& \tilde{G}=\frac{G\left(1+4 \pi r^{3} P / m c^{2}\right)}{1-2 G m / r c^{2}}=\frac{G\left(1+\omega(u) y / c^{2} x\right)}{1-2 G u x / c^{2}}, \\
& \omega=\frac{P^{2}}{u \rho^{2}}=[u]_{1} .
\end{aligned}
$$

\section{PROPOSITION 3}

Let $x(r)$ correspond to an equilibrium $(m(r), P(r)), 0<r<r_{+}$. If there is $r_{0} \in$ $\left(0, r_{+}\right)$such that $x\left(r_{0}\right)>1 / G$, then $r_{+}<+\infty$ and enjoys the estimate

$$
r_{+}<r_{0} \exp \left(\frac{1}{G x\left(r_{0}\right)-1}\right) \text {. }
$$

A proof can be found in the last part of the proof of [7, Theorem 1].

As in [7] we can claim the following result.

\section{PROPOSITION 4}

If $4 / 3<\gamma<2$, then any equilibrium is short.

When $6 / 5<\gamma \leq 4 / 3$, it is known that if $A$ is small and if $P(\rho)$ is sufficiently near to the exact $\gamma$-law $P=A \rho^{\gamma}$, then any equilibrium is short (see [14]). Even if $1<\gamma \leq 6 / 5$, it is possible that there are short equilibria, since Proposition 3 guarantees the existence of tails of short equilibria in any case and we can arbitrarily modify the equation of state in the higher density region. Anyway, in this article we assume Assumptions 1 and 2 only with $1<\gamma<2$ and suppose that a short equilibrium is given to us.

Let us observe roughly the behavior of a short equilibrium $(m(r), P(r))$ at the surface $r=r_{+}$. By Proposition 2 we have $u \in C\left(\left(0, r_{+}\right]\right)$with $u\left(r_{+}\right)=0$, so $P(r), \rho(r)$ are as well. Hence,

$$
r \mapsto m(r)=\int_{0}^{r} 4 \pi r^{\prime 2} \rho\left(r^{\prime}\right) d r^{\prime}
$$

belongs to $C\left(\left(0, r_{+}\right]\right)$. Put

$$
m_{+}=m\left(r_{+}\right)=\int_{0}^{r_{+}} 4 \pi r^{2} \rho(r) d r .
$$


By definition we have $1-2 G m / c^{2} r>0$. Therefore,

$$
\kappa=\lim _{r \rightarrow r_{+}} 1-2 G m / c^{2} r=1-2 G m_{+} / c^{2} r_{+}
$$

is nonnegative. We claim that $\kappa>0$. Otherwise, if $\kappa=0$, then

$$
\frac{d}{d r}\left(1-2 G m / c^{2} r\right)=-\frac{2 G}{c^{2}}\left(4 \pi r \rho-\frac{m}{r^{2}}\right) \rightarrow \frac{2 G m_{+}}{c^{2} r_{+}^{2}}=\frac{1}{r_{+}}
$$

as $r \rightarrow r_{+}-0$ and

$$
1-2 G m / c^{2} r \sim-\frac{1}{r_{+}}\left(r_{+}-r\right),
$$

which contradicts that $1-2 G m / c^{2} r>0$ for $r<r_{+}$. Hence, $\kappa>0$ and

$$
\frac{d u}{d r} \rightarrow-K
$$

as $r \rightarrow r_{+}-0$. Here

$$
K=\frac{G m_{+}}{r_{+}^{2}\left(1-2 G m_{+} / c^{2} r_{+}\right)}
$$

is a positive constant. Hence, since $u \rightarrow 0$ as $r \rightarrow r_{+}$, we see that

$$
u \sim K\left(r_{+}-r\right)
$$

and thus we have the following result.

\section{PROPOSITION 5}

Let $(m(r), P(r)), 0<r<r_{+}$, be a short equilibrium. Then we have

$$
\rho \sim\left(\frac{(\gamma-1) K}{A \gamma}\right)^{\frac{1}{\gamma-1}}\left(r_{+}-r\right)^{\frac{1}{\gamma-1}}
$$

as $r \rightarrow r_{+}-0$, where $K$ is the positive constant given by (3.10).

\section{REMARK}

If $(m(r), P(r)), 0<r<r_{+}$, is a short equilibrium, then for $r \geq r_{+}$we put $\rho=$ $P=0$ (vacuum) and we put

$$
d s^{2}=\left(1-\frac{2 G m_{+}}{c^{2} r}\right) c^{2} d t^{2}-\frac{d r^{2}}{1-\frac{2 G m_{+}}{c^{2} r}}-r^{2}\left(d \theta^{2}+\sin ^{2} \theta d \phi^{2}\right),
$$

which is the Schwarzschild's metric (see [5, p. 301]). Here we must take

$$
F(0)=\frac{1}{2} \log \kappa=\frac{1}{2} \log \left(1-\frac{2 G m_{+}}{c^{2} r_{+}}\right) .
$$

Then the components of the metric are continuously differentiable across $r=r_{+}$.

More precise behavior of the equilibrium at the surface can be given as follows.

\section{PROPOSITION 6}

Assume Assumptions 1 and 2, and let $(m(r), P(r)), 0<r<r_{+}$, be a short equilibrium. If $\frac{\gamma}{\gamma-1}$ is an integer, then $u(r)$ is analytic at $r=r_{+}$. 
Proof

We consider the variables

$$
X=\frac{1}{x}=\frac{u r}{m}, \quad Y=\frac{y}{x^{2}}=\frac{4 \pi r^{4} u^{2} \rho^{2}}{m^{2} P} .
$$

Since $d u / d r<0$, we can take $u$ as the independent variable instead of $r$, and the equations turn out to be

$$
\begin{aligned}
& u \frac{d X}{d u}=\left(1+\frac{1}{\tilde{G}}(-X+\alpha Y)\right) X, \\
& u \frac{d Y}{d u}=\left(2+\beta+\frac{1}{\tilde{G}}(-4 X+2 \alpha Y)\right) Y,
\end{aligned}
$$

where we note

$$
\tilde{G}=G\left(1+\frac{\omega u}{c^{2}} \frac{Y}{X}\right) /\left(1-\frac{2 G}{c^{2}} \frac{u}{X}\right) .
$$

Note that $\tilde{G}>0$ and $\tilde{G} \rightarrow G / \kappa$ as $u \rightarrow+0$, where $\kappa$ is the positive constant given in (3.9). Put

$$
\check{X}=\frac{X}{u}=\frac{r}{m}, \quad \check{Y}=\frac{Y}{u^{\frac{\gamma}{\gamma-1}}}=\frac{4 \pi r^{4} u^{\frac{\gamma-2}{\gamma-1}} \rho^{2}}{m^{2} P} .
$$

We know that $u \mapsto \check{X}$ and $u \mapsto \check{Y}$ belong to $C\left(\left[0, u_{c}\right)\right)$ and $\left.\check{X}\right|_{u=0},\left.\check{Y}\right|_{u=0}$ are positive. Therefore $u \mapsto \tilde{G}=G\left(1+\frac{\omega u^{1 /(\gamma-1)}}{c^{2}} \frac{\check{Y}}{\check{X}}\right) /\left(1-\frac{2 G}{c^{2}} \frac{1}{\check{X}}\right)$ belongs to $C\left(\left[0, u_{c}\right)\right)$.

Integrating (3.11a), we have

$$
X=C_{1} u \exp \left[\int_{0}^{u} \frac{1}{\tilde{G}}\left(-\check{X}+\alpha u^{\frac{\gamma}{\gamma-1}} \check{Y}\right) d u\right] .
$$

Since the integrand is continuous, we see that $u \mapsto \check{X}$ belongs to $C^{1}\left(\left[0, u_{c}\right)\right)$. Integrating (3.11b), we have

$$
Y=C_{2} u^{\frac{\gamma}{\gamma-1}} \exp \left[\int_{0}^{u}\left(\frac{1}{\tilde{G}}\left(-4 \check{X}+2 \alpha u^{\frac{\gamma}{\gamma-1}} \check{Y}\right)+\Omega(u)\right) d u\right]
$$

where

$$
2+\beta=\frac{\gamma}{\gamma-1}+\Omega(u) u, \quad \Omega(u)=[u]_{0} .
$$

Fixing $u_{0}>0$ small, we put $\check{X}_{0}:=\check{X}\left(u_{0}\right), \check{Y}_{0}:=\check{Y}\left(u_{0}\right)$. Since we know that

$$
\check{X}(u) \rightarrow \check{X}_{*}:=\frac{r_{+}}{m_{+}}, \quad \check{Y}(u) \rightarrow \check{Y}_{*}:=4 \pi\left(\frac{\gamma-1}{A \gamma}\right)^{\frac{2-\gamma}{\gamma-1}} \frac{r_{+}^{4}}{m_{+}^{2}}
$$

as $u \rightarrow 0$, we see that if $u_{0}$ is sufficiently small, then $\check{X}_{0}, \check{Y}_{0}$ is arbitrarily near to $\check{X}_{*}, \check{Y}_{*}$, respectively. Now $(\check{X}(u), \check{Y}(u))$ is the unique solution of the integral equation

$$
\begin{aligned}
& \check{X}(u)=\check{X}_{0} \exp \left[-\int_{u}^{u_{0}} \frac{1}{\tilde{G}}\left(-\check{X}+\alpha u^{\frac{\gamma}{\gamma-1}} \check{Y}\right) d u\right] \\
& \check{Y}(u)=\check{Y}_{0} \exp \left[-\int_{u}^{u_{0}}\left(\frac{1}{\tilde{G}}\left(-4 \check{X}+2 \alpha u^{\frac{\gamma}{\gamma-1}} \check{Y}\right)+\Omega(u)\right) d u\right] .
\end{aligned}
$$


Let us denote by $D_{\delta}$ the set $\left\{\left(z_{1}, z_{2}\right) \in \mathbb{C}^{2}|| z_{1}-\check{X}_{*}|<\delta,| z_{2}-\check{Y}_{*} \mid<\delta\right\}$, with $\delta$ being a small positive number. Note that if $|u| \leq \varepsilon_{0}$, with $\varepsilon_{0}$ being a fixed small positive number, and if $(\check{X}, \check{Y}) \in D_{\delta}, 0<\delta \leq \delta_{0}$, then $|1 / \tilde{G}| \leq M_{0}, M_{0}$ depending upon only $\varepsilon_{0}, \delta_{0}$. In fact, since $\check{X}_{*}>0$ and $\delta$ is very small, we can suppose that $(\check{X}, \check{Y}) \in D_{\delta}$ guarantees $|\check{X}| \geq \delta$. Let us consider the functional family $\mathfrak{F}(\varepsilon, \delta)$ of all analytic functions $\left(\phi_{1}(u), \phi_{2}(u)\right)$ defined and analytic for $|u| \leq \varepsilon$ and valued in $D_{\delta}$. The right-hand sides of (3.14a) and (3.14b), in which $\check{X}=\phi_{1}(u)$ and $\check{Y}=\phi_{2}(u)$, will be denoted by $\bar{\phi}_{1}(u)$ and $\bar{\phi}_{2}(u)$, respectively. Then it is easy to see that if $\left|u_{0}\right| \leq \varepsilon$, with $\varepsilon$ being sufficiently small, then $\left(\check{X}_{0}, \check{Y}_{0}\right) \in D_{\delta / 2}$, and if $\left(\phi_{1}, \phi_{2}\right) \in \mathfrak{F}(\varepsilon, \delta)$, then $\left(\bar{\phi}_{1}, \bar{\phi}_{2}\right) \in \mathfrak{F}(\varepsilon, \delta)$. Applying the well-known fixed point theorem (see, e.g., [4, Chapter I, Théorème 7$]$ ), we have a fixed function in $\mathfrak{F}(\varepsilon, \delta)$. This is our $(\check{X}(u), \breve{Y}(u))$ by dint of the uniqueness.

Integrating

$$
\frac{1}{r} \frac{d r}{d u}=-\frac{\check{X}}{\tilde{G}}
$$

we see that $u \mapsto r$ is analytic and $d r / d u<0$ including $u=0$. Hence, the inverse function $r \mapsto u$ is analytic at $r=r_{+}$.

Hereafter we suppose the following.

\section{ASSUMPTION 3}

We assume that $1<\gamma<2$ and that $\frac{\gamma}{\gamma-1}$ is an integer.

Under this assumption, $\frac{1}{\gamma-1}$ is an integer, and since

$$
\rho=\left(\frac{\gamma-1}{A \gamma} u\right)^{\frac{1}{\gamma-1}}\left(1+[u]_{1}\right),
$$

the density distribution $\rho$ of the equilibrium is analytic at $r=r_{+}$, too:

$$
\rho=\left(\frac{(\gamma-1) K}{A \gamma}\right)^{\frac{1}{\gamma-1}}\left(r_{+}-r\right)^{\frac{1}{\gamma-1}}\left(1+\left[r_{+}-r\right]_{1}\right) .
$$

\section{SUPPLEMENTARY REMARK 2}

Here we are considering a short equilibrium with surface $r=r_{+}$and the Schwartzschild's metric in the exterior vacuum region. In other words, the metric

$$
d s^{2}=g_{00} c^{2} d t^{2}+g_{11} d r^{2}-r^{2}\left(d \theta^{2}+\sin ^{2} \theta d \phi^{2}\right)
$$

is given by

$$
\begin{gathered}
g_{00}= \begin{cases}e^{2 F}=\kappa e^{-2 u / c^{2}} & 0 \leq r \leq r_{+}, \\
1-\frac{2 G m_{+}}{c^{2} r} & r_{+}<r,\end{cases} \\
-g_{11}= \begin{cases}e^{2 H}=\left(1-\frac{2 G m}{c^{2} r}\right)^{-1} & 0 \leq r \leq r_{+}, \\
\left(1-\frac{2 G m_{+}}{c^{2} r}\right)^{-1} & r_{+}<r .\end{cases}
\end{gathered}
$$

Let us check that the components $g_{00}, g_{11}$ are of class $C^{2}$ across $r=r_{+}$. 
It is clear that $g_{00}$ and $g_{11}$ are continuous since $u \rightarrow 0, m \rightarrow m_{+}$as $r \rightarrow r_{+}-0$ and $\kappa=1-2 G m_{+} / c^{2} r_{+}$. Moreover, we have

$$
\left.\frac{d}{d r} g_{00}\right|_{r=r_{+}-0}=-\left.\frac{2 \kappa}{c^{2}} \frac{d u}{d r}\right|_{r=r_{+}-0}=\frac{2 G m_{+}}{c^{2} r_{+}^{2}},
$$

since $d u / d r \rightarrow-K$ with $K$ given by (3.10), and

$$
\left.\frac{d^{2}}{d x^{2}} g_{00}\right|_{r=r_{+}-0}=\frac{4 \kappa}{c^{4}}\left(\frac{d u}{d r}\right)_{r=r_{+}-0}^{2}-\left.\frac{2 \kappa}{c^{2}} \frac{d^{2} u}{d r^{2}}\right|_{r=r_{+}-0}=-\frac{4 G m_{+}}{c^{2} r_{+}^{3}} .
$$

This can be verified by differentiating (3.3) and seeing

$$
\left.\frac{d^{2} u}{d r^{2}}\right|_{r=r_{+}-0}=\frac{2 G m_{+}}{r_{+}^{3} \kappa}+\frac{2}{c^{2}}\left(\frac{G m_{+}}{r_{+}^{2} \kappa}\right)^{2} .
$$

Hence, $g_{00}$ is twice continuously differentiable at $r=r_{+}$. On the other hand, it is easy to see that the patched function

$$
\tilde{m}(r)= \begin{cases}m(r) & 0 \leq r \leq r_{+} \\ m_{+} & r_{+}<r\end{cases}
$$

is of class $C^{k}$ if and only if $\gamma<k /(k-1)$ since

$$
\frac{d m}{d r}=4 \pi \rho r^{2}=4 \pi r_{+}^{2}\left(\frac{(\gamma-1) K}{A \gamma}\right)^{1 /(\gamma-1)}\left(r_{+}-r\right)^{\frac{1}{\gamma-1}}\left(1+\left[r_{+}-r\right]_{1}\right) .
$$

Hence $\tilde{m}$ and $g_{11}$ are of class $C^{2}$ since $\gamma<2$.

\section{Equations for perturbations}

Let us fix a short equilibrium $\rho(r)$ which is positive on $0 \leq r<r_{+}$. Put $m_{+}=$ $m\left(r_{+}\right)$. Then we can take $m$ as an independent variable and get an equilibrium $\rho=\bar{\rho}(m)$ and $r=r(m), 0 \leq m \leq m_{+}$. We have to consider solutions of $\left(E_{c}\right)$ near this equilibrium of the form

$$
\begin{aligned}
& R=r(m)(1+y), \\
& V=r(m) v .
\end{aligned}
$$

Here $y$ and $v$ are small perturbations. The equations turn out to be

$$
\begin{aligned}
e^{-F} \frac{\partial y}{\partial t}= & \left(1+\frac{P}{c^{2} \rho}\right) v \\
e^{-F} \frac{\partial v}{\partial t}= & \frac{4 \pi}{c^{2}} r^{2}(1+y)^{2} P v \frac{\partial}{\partial m}(r v) \\
& -\frac{G}{r^{3}(1+y)^{2}}\left(m+\frac{4 \pi}{c^{2}} \operatorname{Pr}^{3}(1+y)^{3}\right) \\
& -\left(1+\frac{r^{2} v^{2}}{c^{2}}-\frac{2 G m}{c^{2} r(1+y)}\right)\left(1+\frac{P}{c^{2} \rho}\right)^{-1} \cdot 4 \pi r(1+y)^{2} \frac{\partial P}{\partial m}
\end{aligned}
$$

Instead of $m$, let us take $r=r(m)$ as the independent variable. Since

$$
\frac{d m}{d r}=4 \pi \bar{\rho} r^{2}
$$


we see

$$
\frac{\partial}{\partial m}=\frac{1}{4 \pi \bar{\rho} r^{2}} \frac{\partial}{\partial r}
$$

Therefore, (2.24) and (4.1) imply

$$
\rho=\bar{\rho}(1+y)^{-2}\left(1+y+r \frac{\partial y}{\partial r}\right)^{-1}
$$

so that

$$
\rho=\bar{\rho}\left(1-3 y-r \frac{\partial y}{\partial r}+\left[y, r \frac{\partial y}{\partial r}\right]_{2}\right) .
$$

Here $\left[X_{1}, X_{2}\right]_{2}$ denotes a convergent double power series of the form

$$
\sum_{k_{1}+k_{2} \geq 2} a_{k_{1} k_{2}} X_{1}^{k_{1}} X_{2}^{k_{2}}
$$

Let us recall that $(\bar{\rho})^{\gamma-1} \in C^{\infty}\left(\left[0, r_{+}\right]\right)$, provided that $\gamma /(\gamma-1)$ is an integer, say, from Assumption 3.

Then (4.4) reads

$$
\begin{aligned}
e^{-F} \frac{\partial v}{\partial t}= & \frac{1}{c^{2}}(1+y)^{2} \frac{P}{\bar{\rho}} v \frac{\partial}{\partial r}(r v) \\
& -\frac{G}{r^{3}(1+y)^{2}}\left(m+\frac{4 \pi}{c^{2}} \operatorname{Pr}^{3}(1+y)^{3}\right) \\
& -\left(1+\frac{r^{2} v^{2}}{c^{2}}-\frac{2 G m}{c^{2} r(1+y)}\right)\left(1+\frac{P}{c^{2} \rho}\right)^{-1} \frac{(1+y)^{2}}{r \bar{\rho}} \frac{\partial P}{\partial r} .
\end{aligned}
$$

We have to solve (4.3)-(4.6) for unknown functions $(t, r) \mapsto y, v$, where $r$ is confined to the fixed interval $\left[0, r_{+}\right]$. Here $m=m(r)$ is determined by the equilibrium through

$$
m=4 \pi \int_{0}^{r} \bar{\rho}(r) r^{2} d r
$$

and $\rho, P(\rho), u(\rho)$ are given functions of $\bar{\rho}(r)$ and the unknowns $y, r \partial y / \partial r$ through (4.5).

The perturbation of $\rho$ is expressed by (4.5). Similar expressions of $P$ and $u$ are necessary. If $P(\rho)$ was the exact $\gamma$-law, say, if $P=A \rho^{\gamma}$, then we would have

$$
\begin{aligned}
P & =\bar{P}(1+y)^{-2 \gamma}\left(1+y+r \frac{\partial y}{\partial r}\right)^{-\gamma} \\
& =\bar{P}\left(1-\gamma\left(3 y+r \frac{\partial y}{\partial r}\right)+\left[y, r \frac{\partial y}{\partial r}\right]_{2}\right) .
\end{aligned}
$$

However, this exact $\gamma$-law is not treated by this article, since it violates the condition $d P / d \rho<c^{2}$ for large $\rho$. Our case should be treated more carefully.

We should introduce the quantity

$$
\gamma^{P}:=\frac{\rho}{P} \frac{d P}{d \rho}
$$


Then under the Assumption 2 we see that

$$
\gamma^{P}=\gamma+[u]_{1},
$$

and using this function, we can express

$$
P=\bar{P}\left(1-\gamma^{P}(\bar{u})\left(3 y+r \frac{\partial y}{\partial r}\right)-\Phi^{P}\left(\bar{u}, y, r \frac{\partial y}{\partial r}\right)\right),
$$

where

$$
\Phi^{P}\left(u, y, r y^{\prime}\right)=\left[u ; y, r y^{\prime}\right]_{0 ; 2} .
$$

Here $\left[X_{0} ; X_{1}, X_{2}\right]_{0 ; 2}$ denotes a convergent triple power series of the form

$$
\sum_{k_{0} \geq 0, k_{1}+k_{2} \geq 2} a_{k_{0} k_{1} k_{2}} X_{0}^{k_{0}} X_{1}^{k_{1}} X_{2}^{k_{2}} .
$$

We note that

$$
\begin{aligned}
\left(1+\frac{P}{c^{2} \rho}\right)^{-1}= & \left(1+\frac{\bar{P}}{c^{2} \bar{\rho}}\right)^{-1}\left(1+\frac{\bar{P}}{c^{2} \bar{\rho}}\left(1+\frac{\bar{P}}{c^{2} \bar{\rho}}\right)^{-1}\left(\gamma^{P}-1\right)\left(3 y+r \frac{\partial y}{\partial r}\right)\right. \\
& \left.+\left[\bar{u} ; y, r \frac{\partial y}{\partial r}\right]_{0 ; 2}\right)
\end{aligned}
$$

\section{SUPPLEMENTARY REMARK 3}

The letter $r$ is used, on the one hand, as one of the comoving coordinates for the metric (2.5) and, on the other hand, as one of the independent variables of (4.6). However, these two quantities denoted by the same letter $r$ do not coincide if we consider moving solutions. Therefore, in order to clarify the relation between these two quantities, we shall denote by $r^{*}$ the latter $r$, that is, one of the independent variables for (4.6).

In other words, the definition of $r^{*}=\varphi(t, r)$ is as follows. Put

$$
m=f_{1}(r):=4 \pi \int_{0}^{r} \bar{\rho}\left(r^{\prime}\right) r^{\prime 2} d r^{\prime} \quad\left(0 \leq r \leq r_{+}\right)
$$

along the equilibrium fixed. Then we have the inverse function $r=f_{1}^{-1}(m)$ defined on $0 \leq m \leq m_{+}$. But along the moving solutions, $m$ is one of the variables of (2.25)-(2.26) or $\left(E_{c}\right)$ defined by (2.11). So we denote

$$
m=f_{2}(t, r):=4 \pi \int_{0}^{r} \rho\left(t, r^{\prime}\right) R\left(t, r^{\prime}\right)^{2} \partial_{r} R\left(t, r^{\prime}\right) d r^{\prime}
$$

along the moving solutions under consideration. Then we put

$$
r^{*}=\varphi(t, r):=f_{1}^{-1}\left(f_{2}(t, r)\right) .
$$

Let us determine the function $\varphi(t, x)$. The function $m=f_{2}(t, r)$ should satisfy (2.20d); that is,

$$
e^{-F} \frac{\partial m}{\partial t}=-\frac{4 \pi}{c^{2}} R P V .
$$


The left-hand side of (4.10) is

$$
\frac{1}{\sqrt{\kappa}} e^{u / c^{2}} D f_{1}\left(r^{*}\right) \frac{\partial r^{*}}{\partial t}=\frac{1}{\sqrt{\kappa}} e^{u / c^{2}} \cdot 4 \pi \bar{\rho}\left(r^{*}\right)\left(r^{*}\right)^{2} \frac{\partial r^{*}}{\partial t} .
$$

On the other hand, we are going to construct moving solutions of the form

$$
\begin{aligned}
& R=r^{*}\left(1+y\left(t, r^{*}\right)\right), \quad V=r^{*} v\left(t, r^{*}\right), \\
& P=P(\rho), \quad \rho=\bar{\rho}\left(r^{*}\right)\left(1+y\left(t, r^{*}\right)\right)^{-2}\left(1+y+r^{*} \frac{\partial y}{\partial r^{*}}\right)^{-1}
\end{aligned}
$$

with $y, v \in C^{\infty}\left([0, T] \times\left[0, r_{+}\right]\right)$, which are very small. Suppose that we have constructed such solutions. Then the function $\varphi(t, r)$ should satisfy

$$
\frac{\partial}{\partial t} \varphi(t, r)=-\frac{\sqrt{\kappa}}{c^{2}} e^{-u / c^{2}}(1+y)^{2} \frac{P}{\bar{\rho}} v \cdot \varphi(t, r),
$$

where $u=u(\rho), y, P=P(\rho), \bar{\rho}, v$ on the right-hand side are evaluated at $\left(t, r^{*}\right)=(t, \varphi(t, r))$. The formula $(4.11)$ can be considered as an ordinary differential equation for $\varphi(\cdot, r)$ for each fixed $r$, which determines $\varphi(\cdot, r)$ provided that the initial value $\varphi(0, r)=f_{1}^{-1}\left(f_{2}(0, r)\right)$ is given.

But we can assume that $\varphi(0, r)=r$ without loss of generality. In fact, $\varphi(0, r)=r$ means $f_{1}(r)=f_{2}(0, r)$, and even if $f_{1} \neq f_{2}(0, \cdot)$, we can find the change of variable $r=\psi\left(r^{b}\right)$ such that $f_{1}\left(r^{b}\right)=f_{2}\left(0, \psi\left(r^{b}\right)\right)$. Considering $r^{b}$ instead of $r$, we can assume $f_{1}(r)=f_{2}(0, r)$ or $\varphi(0, r)=r$. Clearly the $C^{\infty}$-solution $\varphi$ is uniquely determined and $\varphi(t, r)-r$ is very small with its derivatives. Of course, $\varphi(t, 0)=0$ and $\varphi\left(t, r_{+}\right)=r_{+}$since $P / \bar{\rho}$ vanishes at $r=r_{+}-0$. Hence, we have the solutions

$$
R=\varphi(t, r)(1+y(t, \varphi(t, r))), \quad V=\varphi(t, r) v(t, \varphi(t, r))
$$

and so on as functions of the original comoving coordinates $t, r$.

\section{Analysis of the linearized equation}

We are going to analyze the linearized equations for (4.3)-(4.6) and establish the existence of time-periodic solutions to the linearized equations of the form

$$
y=\text { const } \sin (\sqrt{\lambda} t+\text { const }) \tilde{\psi}(r),
$$

where $\lambda>0$ and $\tilde{\psi}(r)$ is an analytic function of $r$ in a neighborhood of $\left[0, r_{+}\right]$.

Using the formulas listed in the last part of the preceding section, we see that the linearizations of (4.3)-(4.6) turn out to be

$$
\begin{aligned}
& e^{-F} \frac{\partial y}{\partial t}=\left(1+\frac{P}{c^{2} \rho}\right) v, \\
& e^{-F} \frac{\partial v}{\partial t}=E_{2} y^{\prime \prime}+E_{1} y^{\prime}+E_{0} y,
\end{aligned}
$$

where $y^{\prime \prime}=\partial^{2} y / \partial r^{2}, y^{\prime}=\partial y / \partial r$, and

$$
E_{2}=e^{-2 H}\left(\rho+P / c^{2}\right)^{-1} \gamma^{P} P
$$




$$
\begin{aligned}
\frac{E_{1}}{E_{2}}= & \frac{4 \pi G}{c^{2}} e^{2 H}\left(\rho+P / c^{2}\right) r-\left(\rho+P / c^{2}\right)^{-1}\left(1-\frac{1}{\gamma_{p}}\right) \frac{P^{\prime}}{c^{2}}+\frac{3}{r}+\frac{\left(\gamma^{P} P r\right)^{\prime}}{\gamma^{P} P r} \\
= & F^{\prime}+H^{\prime}-\frac{\left(1+P / c^{2} \rho\right)^{\prime}}{1+P / c^{2} \rho}+\frac{3}{r}+\frac{\left(\gamma^{P} P r\right)^{\prime}}{\gamma^{P} P r} \\
E_{0}= & \frac{4 \pi G}{c^{2}} \cdot 3\left(\gamma^{P}-1\right) P \\
& +\left(-1-3 \gamma^{P} e^{-2 H}+3\left(\gamma^{P}-1\right) e^{-2 H}\left(1+P / c^{2} \rho\right)^{-1}\right)\left(\rho+P / c^{2}\right)^{-1} \frac{P^{\prime}}{r} \\
& +3 e^{-2 H}\left(\rho+P / c^{2}\right)^{-1} \frac{\left(\gamma^{P} P\right)^{\prime}}{r} .
\end{aligned}
$$

Here $\rho, P, \gamma^{P}, F, H$ are abbreviations for the quantities $\bar{\rho}(r), \bar{P}=P(\bar{\rho}(r))$, $\gamma^{P}(\bar{u}(r)), \bar{F}=F(\bar{u}(r))=-\frac{1}{c^{2}} \bar{u}(r)+\frac{1}{2} \log \kappa, \bar{H}=-\frac{1}{2} \log \left(1-\frac{2 G m}{c^{2} r}\right)$ along the considered equilibrium. Throughout the above manipulations we have used the equation

$$
\frac{4 \pi G}{c^{2}} e^{2 H}\left(\rho+P / c^{2}\right) r=F^{\prime}+H^{\prime},
$$

which can be derived from the differentiation of

$$
e^{-2 H}=1-\frac{2 G m}{c^{2} r}
$$

and $(3.1 \mathrm{~b})$, and also the relation

$$
\frac{\left(1+P / c^{2} \rho\right)^{\prime}}{1+P / c^{2} \rho}=\frac{1}{\rho+P / c^{2}}\left(1-\frac{1}{\gamma^{P}}\right) \frac{P^{\prime}}{c^{2}} .
$$

In other words, the linearized second-order single equation is

$$
\frac{\partial^{2} y}{\partial t^{2}}+\mathcal{L} y=0
$$

where

$$
\begin{aligned}
\mathcal{L} y & =-\frac{a}{b} y^{\prime \prime}-\frac{a^{\prime}}{b} y^{\prime}+Q y=-\frac{1}{b}\left(a y^{\prime}\right)^{\prime}+Q y, \\
a & =\exp \left[\int^{r} \frac{E_{1}}{E_{2}} d r\right]=\frac{\gamma^{P} P r^{4}}{1+P / c^{2} \rho} e^{F+H}, \\
b & =\left(1+P / c^{2} \rho\right)^{-1} \rho r^{4} e^{-F+3 H}, \\
Q & =-e^{2 F}\left(1+P / c^{2} \rho\right) E_{0} .
\end{aligned}
$$

In order to investigate the spectral property of the second-order linear differential operator $\mathcal{L}$, we reduce the eigenvalue problem

$$
\mathcal{L} y=\lambda y
$$

to the normal form

$$
-\frac{d^{2} \eta}{d \xi^{2}}+q(\xi) \eta=\lambda \eta
$$


by the Liouville transformation

$$
\begin{aligned}
\xi & =\int_{0}^{r} \sqrt{\frac{b}{a}} d r=\int_{0}^{r} \sqrt{\frac{\rho}{\gamma^{P} P}} e^{-F+H} d r \\
\eta & =(a b)^{1 / 4} y=\left(\gamma^{P} \rho P\right)^{1 / 4} r^{2}\left(1+P / c^{2} \rho\right)^{-1 / 2} e^{H} y,
\end{aligned}
$$

when the result is

$$
q=Q+\frac{a}{4 b}\left(\left(\frac{a^{\prime}}{a}+\frac{b^{\prime}}{b}\right)^{\prime}-\frac{1}{4}\left(\frac{a^{\prime}}{a}+\frac{b^{\prime}}{b}\right)^{2}+\frac{a^{\prime}}{a}\left(\frac{a^{\prime}}{a}+\frac{b^{\prime}}{b}\right)\right) .
$$

See $[1$, p. 275, Theorem 6].

Since

$$
\sqrt{\frac{\rho}{\gamma^{P} P}} \sim \operatorname{const}\left(r_{+}-r\right)^{-1 / 2}
$$

we can define the finite value

$$
\xi_{+}:=\int_{0}^{r_{+}} \sqrt{\frac{\rho}{\gamma^{P} P}} e^{-F+H} d r .
$$

The interval $\left(0, r_{+}\right)$is mapped onto $\left(0, \xi_{+}\right)$.

First let us observe the behavior of $q$ as $\xi \rightarrow 0(r \rightarrow 0)$. We see that $Q=O(1)$,

$$
\frac{a^{\prime}}{a} \sim \frac{4}{r}, \quad \frac{a^{\prime}}{a}+\frac{b^{\prime}}{b} \sim \frac{8}{r}, \quad\left(\frac{a^{\prime}}{a}+\frac{b^{\prime}}{b}\right)^{\prime} \sim-\frac{8}{r^{2}} .
$$

Therefore,

$$
\left.q \sim 2 \gamma^{P} P \rho^{-1} e^{2 F-2 H}\right|_{r=0} \frac{1}{r^{2}} .
$$

On the other hand we have

$$
\xi \sim\left(\left.\gamma^{P} P \rho^{-1} e^{2 F-2 H}\right|_{r=0}\right)^{-1 / 2} r .
$$

Hence, we have

$$
q \sim \frac{2}{\xi^{2}}
$$

Note that $2>3 / 4$.

Next we observe the behavior of $q$ as $\xi \rightarrow \xi_{+}\left(r \rightarrow r_{+}\right)$. Note that $P^{\prime} / \rho \rightarrow-K$, where $K$ is the constant defined by (3.10). Therefore, we see that $Q=O(1)$. Moreover, we have

$$
\frac{\rho}{\gamma^{P}} \frac{d}{d \rho} \gamma^{P}=O(u) \rightarrow 0,
$$

so that $\left(\gamma^{P}\right)^{\prime} / \gamma^{P}=o\left(\rho^{\prime} / \rho\right)$. Hence, we see that

$$
\begin{aligned}
\frac{a^{\prime}}{a} & \sim-\frac{\gamma}{\gamma-1} \frac{1}{r_{+}-r}, \quad \frac{a^{\prime}}{a}+\frac{b^{\prime}}{b} \sim-\frac{\gamma+1}{\gamma-1} \frac{1}{r_{+}-r}, \\
\left(\frac{a^{\prime}}{a}+\frac{b^{\prime}}{b}\right)^{\prime} & \sim-\frac{\gamma+1}{\gamma-1} \frac{1}{\left(r_{+}-r\right)^{2}} .
\end{aligned}
$$


Therefore, we have

$$
\left.q \sim K e^{2 F-2 H}\right|_{r=r_{+}} \frac{(\gamma+1)(3-\gamma)}{16(\gamma-1)} \frac{1}{r_{+}-r}
$$

On the other hand, we have

$$
\xi_{+}-\left.\xi \sim \frac{2}{\sqrt{(\gamma-1) K}} e^{-F+H}\right|_{r=r_{+}} \sqrt{r_{+}-r} .
$$

Hence, we have

$$
q \sim \frac{(\gamma+1)(3-\gamma)}{4(\gamma-1)^{2}} \frac{1}{\left(\xi_{+}-\xi\right)^{2}} .
$$

It follows from $1<\gamma<2$ that

$$
\frac{(\gamma+1)(3-\gamma)}{4(\gamma-1)^{2}}>\frac{3}{4}
$$

Therefore, both boundary points $\xi=0$ and $\xi_{+}$are of limit point type, and [13, p. 159, Theorem X.10] gives the following conclusion, which is the same as $[8$, Proposition 1].

\section{PROPOSITION 7}

The operator $\mathfrak{T}_{0}, \mathcal{D}\left(\mathfrak{T}_{0}\right)=C_{0}^{\infty}\left(0, \xi_{+}\right), \mathfrak{T}_{0} \eta=-\eta_{\xi \xi}+q \eta$, in $L^{2}\left(0, \xi_{+}\right)$has the Friedrichs extension $\mathfrak{T}$, a self-adjoint operator, whose spectrum consists of simple eigenvalues $\lambda_{1}<\cdots<\lambda_{n}<\cdots \rightarrow+\infty$. In other words, the operator $\mathfrak{S}_{0}, \mathcal{D}\left(\mathfrak{S}_{0}\right)=$ $C_{0}^{\infty}\left(0, r_{+}\right), \mathfrak{S}_{0} y=\mathcal{L} y$ in $L^{2}\left(\left(0, r_{+}\right), b d r\right)$ has the Friedrichs extension $\mathfrak{S}$, a selfadjoint operator with eigenvalues $\left(\lambda_{n}\right)_{n}$.

In order to investigate the structure of the linear operator $\mathcal{L}$, we introduce the new independent variable $x$ instead of $r$ defined by

$$
x:=\frac{\tan ^{2} \theta}{1+\tan ^{2} \theta} \quad \text { with } \theta:=\frac{\pi \xi}{2 \xi_{+}}=\frac{\pi}{2 \xi_{+}} \int_{0}^{r} \sqrt{\frac{\rho}{\gamma^{P} P}} e^{-F+H} d r .
$$

The interval $\left[0, r_{+}\right]$of the variable $r$ is mapped onto $[0,1]$ of $x$, and we have

$$
\begin{aligned}
\frac{d}{d r}= & \frac{\pi}{\xi_{+}} \sqrt{x(1-x)} \sqrt{\frac{b}{a}} \frac{d}{d x}, \\
\frac{d^{2}}{d r^{2}}= & \left(\frac{\pi}{\xi_{+}}\right)^{2} \frac{b}{a}\left(x(1-x) \frac{d^{2}}{d x^{2}}+\left(\frac{1-2 x}{2}\right.\right. \\
& \left.\left.+\frac{\xi_{+}}{\pi} \sqrt{x(1-x)} \sqrt{\frac{a}{b}} \frac{1}{2} \frac{a}{b} \frac{d}{d r}\left(\frac{b}{a}\right)\right) \frac{d}{d x}\right) .
\end{aligned}
$$

We note

$$
r \frac{d}{d r}=x[(x)] \frac{d}{d x},
$$

where and hereafter $[(x)]$ denotes an analytic function of $x$ in a neighborhood of the interval $[0,1]$. In fact, (5.12a) implies the following observations. As $r \rightarrow 0$ 
$(x \rightarrow 0)$, we see

$$
r=\frac{\xi_{+}}{\pi} C_{0} \sqrt{x}\left(1+[x]_{1}\right) \quad \text { with } C_{0}=\left.2 \sqrt{\frac{\gamma^{P} P}{\rho}} e^{F-H}\right|_{r=0},
$$

and

$$
\sqrt{\frac{b}{a}}=\frac{2 \xi_{+}}{\pi} \frac{\sqrt{x}}{r}\left(1+[x]_{1}\right)
$$

so that

$$
r \frac{d}{d r}=2 x\left(1+[x]_{1}\right) \frac{d}{d x} .
$$

As $r \rightarrow r_{+}(x \rightarrow 1)$, we have

$$
1-x=\left(\frac{\pi}{\xi_{+}}\right)^{2} C_{1}\left(r_{+}-r\right)\left(1+\left[r_{+}-r\right]_{1}\right) \quad \text { with } C_{1}=\frac{1}{(\gamma-1) \kappa^{2} K}
$$

(see (3.16) and note $e^{F-H}=\kappa+\left[r_{+}-r\right]_{1}$ with $\left.\kappa=1-2 G m_{+} / c^{2} r_{+}\right)$and

$$
\sqrt{\frac{b}{a}}=\frac{\pi}{\xi_{+}} \frac{C_{1}}{\sqrt{1-x}}\left(1+[1-x]_{1}\right),
$$

so that

$$
r \frac{d}{d r}=\left(\frac{\pi}{\xi_{+}}\right)^{2} C_{1} r_{+}\left(1+[1-x]_{1}\right) \frac{d}{d x} .
$$

Now we can write

$$
\left(\frac{\xi_{+}}{\pi}\right)^{2} \mathcal{L} y=-x(1-x) \frac{d^{2}}{d x^{2}}-B \frac{d y}{d x}+\left(\frac{\xi_{+}}{\pi}\right)^{2} Q y,
$$

where

$$
B=\frac{1-2 x}{2}+\frac{\xi_{+}}{\pi} \sqrt{x(1-x)} \sqrt{\frac{a}{b}}\left(\frac{1}{2} \frac{a}{b} \frac{d}{d r}\left(\frac{b}{a}\right)+\frac{1}{a} \frac{d a}{d r}\right) .
$$

- As $r \rightarrow 0(x \rightarrow 0)$, we see

$$
B=\frac{5}{2}+[x]_{1} .
$$

We have

$$
\begin{aligned}
\frac{1}{2} \frac{a}{b} \frac{d}{d r}\left(\frac{b}{a}\right)+\frac{1}{a} \frac{d a}{d r} & =\frac{1}{2} \frac{\left(\gamma^{P} P \rho\right)^{\prime}}{\gamma^{P} P \rho}+2 H^{\prime}+\frac{4}{r}-\frac{\left(1+P / c^{2} \rho\right)^{\prime}}{1+P / c^{2} \rho} \\
& =\frac{4}{r}\left(1+\left[r^{2}\right]_{1}\right)
\end{aligned}
$$

and

$$
\begin{aligned}
\sqrt{\frac{a}{b}} & =\sqrt{\frac{\gamma^{P} P}{\rho}} e^{F-H} \\
& =\left.\left(\sqrt{\frac{\gamma^{P} P}{\rho}} e^{F-H}\right)\right|_{r=0}\left(1+\left[r^{2}\right]_{1}\right)=\frac{\pi}{2 \xi_{+}} \frac{r}{\sqrt{x}}\left(1+[x]_{1}\right) .
\end{aligned}
$$


Clearly

$$
Q=e^{-F}\left(1+P / c^{2} \rho\right) E_{0}=\left[r^{2}\right]_{0}=[x]_{0} .
$$

- As $r \rightarrow r_{+}(x \rightarrow 1)$, we see

$$
B=-\frac{\gamma}{\gamma-1}+[1-x]_{1}
$$

We have

$$
\begin{aligned}
\frac{1}{2} \frac{a}{b} \frac{d}{d r}\left(\frac{b}{a}\right)+\frac{1}{a} \frac{d a}{d r} & =-\frac{1}{2} \frac{\gamma+1}{\gamma-1} \frac{1}{r_{+}-r}\left(1+\left[r_{+}-r\right]_{1}\right) \\
& =-\frac{\gamma+1}{\gamma-1}\left(\frac{\pi}{\xi_{+}}\right)^{2} \frac{C_{1}}{1-x}\left(1+[1-x]_{1}\right)
\end{aligned}
$$

and

$$
\frac{a}{b}=\frac{1}{C_{1}}\left(r_{+}-r\right)\left(1+\left[r_{+}-r\right]_{1}\right)=\frac{1}{C_{1}^{2}}\left(\frac{\xi_{+}}{\pi}\right)^{2}(1-x)\left(1+[1-x]_{1}\right) .
$$

Clearly

$$
Q=[u]_{0}=\left[r_{+}-r\right]_{0}=[1-x]_{0} .
$$

In summary, we have the following conclusion, which is the same as $[8$, Proposition 3].

\section{PROPOSITION 8}

We can write

$$
\left(\frac{\xi_{+}}{\pi}\right)^{2} \mathcal{L} y=-x(1-x) \frac{d^{2} y}{d x^{2}}-\left(\frac{5}{2}(1-x)-\frac{N}{2} x\right) \frac{d y}{d x}+L_{1}(x) \frac{d y}{d x}+L_{0}(x) y,
$$

where $L_{1}(x)=x(1-x)[(x)], L_{0}(x)=[(x)]$. Here $N$ is the parameter defined by

$$
N=\frac{2 \gamma}{\gamma-1} \quad \text { or } \quad \gamma=\frac{N}{N-2} .
$$

Assumption 3 reads that $N$ is an even integer greater than 4 . As long as we are concerned with investigating the analytic structure of the operator $\mathcal{L}$, we may assume that $\xi_{+}=\pi$ without loss of generality.

\section{PROPOSITION 9}

Let $\lambda=\lambda_{n}$ be a positive eigenvalue, and let $\psi$ be an associated eigenfunction which belongs to $L^{2}\left([0,1] ; x^{\frac{3}{2}}(1-x)^{\frac{N}{2}-1} d x\right)$. Then

$$
Y_{1}=\sin \left(\sqrt{\lambda} t+\Theta_{0}\right) \psi(x)
$$

is a time-periodic solution of the linearized problem (5.3).

Thanks to Proposition 8, we can claim the following proposition on the analytic property of the eigenfunction, which is the same as [8, Proposition 4]. 
PROPOSITION 10

We have

$$
\begin{aligned}
& \psi(x)=c_{0}\left(1+[x]_{1}\right) \quad \text { as } x \rightarrow 0, \\
& \psi(x)=c_{1}\left(1+[1-x]_{1}\right) \quad \text { as } x \rightarrow 1 .
\end{aligned}
$$

Here $c_{0}, c_{1}$ are nonzero constants. Other independent solutions of $\mathcal{L} y=\lambda y$ do not belong to $L^{2}\left([0,1] ;(1-x)^{\frac{N}{2}-1} d x\right)$ as $x \sim 1$.

Therefore $\psi(x)=[(x)]$, and $Y_{1}$ is an analytic function of $t \in \mathbb{C}$ and $x$ on a neighborhood of $[0,1]$ independent of $t$. Hereafter we fix such a time-periodic function $Y_{1}$.

\section{Rewriting of the equations (4.3)-(4.6) using the linear operator $\mathcal{L}$}

Let us go back to the system of equations (4.3)-(4.6). In order to rewrite these equations using the linear operator $\mathcal{L}$, we shall use the following observations.

We are considering the perturbed $P$ such that

$$
P=\bar{P}\left(1-\gamma^{P}(\bar{u})(3 y+z)-\Phi^{P}(\bar{u}, y, z)\right),
$$

where $z=r \partial y / \partial r$. Then we have

$$
\begin{aligned}
-\frac{1}{r \bar{\rho}} \frac{\partial P}{\partial r}= & -\frac{1}{r \bar{\rho}} \frac{d \bar{P}}{d r}+\left(1+\frac{1}{\gamma^{P}} \partial_{z} \Phi^{P}\right) \frac{1}{r \bar{\rho}} \frac{\partial}{\partial r}\left(\bar{P} \gamma^{P}(3 y+z)\right) \\
& +\frac{\bar{P}}{r \bar{\rho}} \cdot[Q 0]+\frac{1}{r \bar{\rho}} \frac{d \bar{P}}{d r} \cdot[Q 1],
\end{aligned}
$$

where

(6.3a) $[Q 0]:=2\left(\gamma^{P}+\partial_{z} \Phi^{P}\right)(1+y)^{-1} \frac{z^{2}}{r}+\frac{d \bar{u}}{d r}\left(\partial_{u} \Phi^{P}-\frac{1}{\gamma^{P}} \frac{d \gamma^{P}}{d u}(3 y+z) \partial_{z} \Phi^{P}\right)$,

(6.3b) $[Q 1]:=\Phi^{P}-(3 y+z) \partial_{z} \Phi^{P}$.

Here we have used the relation

$$
\left(\partial_{y}-3 \partial_{z}\right) \Phi^{P}=2\left(\gamma^{P}+\partial_{z} \Phi^{P}\right)(1+y)^{-1} z .
$$

Let us analyze

$$
\text { the right-hand side of }(4.6)=[R 1]+[R 2],
$$

where

$$
\begin{aligned}
{[R 1]:=} & -G(1+y)\left(\frac{m}{r^{3}(1+y)^{3}}+\frac{4 \pi P}{c^{2}}\right) \\
& -\left(1+\frac{r^{2} v^{2}}{c^{2}}-\frac{2 G m}{c^{2} r(1+y)}\right)\left(1+P / c^{2} \rho\right)^{-1} \frac{(1+y)^{2}}{r \bar{\rho}} \frac{\partial P}{\partial r}
\end{aligned}
$$

(6.6b) $\quad[R 2]:=\frac{1}{c^{2}}(1+y)^{2} \frac{P}{\bar{\rho}}\left(v^{2}+v w\right) \quad$ with $w=r \frac{\partial v}{\partial r}$. 
Let us put

$$
[R 1]=[R 3]+[R 4]+[R 5]+[R 6]+[R 7]
$$

where

$$
\begin{aligned}
& {[R 3]:=-G(1+y)\left(\frac{m}{r^{3}(1+y)^{3}}+\frac{4 \pi P}{c^{2}}\right)} \\
& =-\frac{G m}{r^{3}}-\frac{4 \pi G \bar{P}}{c^{2}}+[R 3 L]+[R 3 Q], \\
& {[R 3 L]:=\frac{G m}{r^{3}} \cdot 2 y+\frac{4 \pi G}{c^{2}} \bar{P} \gamma^{P}(3 y+z)+\frac{4 \pi G}{c^{2}} \bar{P} y,} \\
& {[R 3 Q]:=-\frac{G m}{r^{3}}\left(\frac{1}{(1+y)^{2}}-(1-2 y)\right)+\frac{4 \pi G}{c^{2}} \bar{P} \Phi^{P}+\frac{4 \pi G}{c^{2}}(P-\bar{P}) y,} \\
& {[R 4]:=-\left(1+\frac{r^{2} v^{2}}{c^{2}}-\frac{2 G m}{c^{2} r(1+y)}\right)\left(1+P / c^{2} \rho\right)^{-1} \frac{(1+y)^{2}}{r \bar{\rho}} \frac{d \bar{P}}{d r}} \\
& =-\left(1-\frac{2 G m}{c^{2} r}\right)\left(1+\bar{P} / c^{2} \bar{\rho}\right)^{-1} \frac{1}{r \bar{\rho}} \frac{d \bar{P}}{d r}+[R 4 L]+[R 4 Q], \\
& {[R 4 L]:=\left(-\frac{2 G m}{c^{2} r}\left(1+\bar{P} / c^{2} \bar{\rho}\right)^{-1} \cdot y\right.} \\
& +\left(1-\frac{2 G m}{c^{2} r}\right) \frac{\bar{P}}{c^{2} \bar{\rho}}\left(1+\bar{P} / c^{2} \rho\right)^{-1}\left(\gamma^{P}-1\right)(3 y+z) \\
& \left.+\left(1-\frac{2 G m}{c^{2} r}\right)\left(1+\bar{P} / c^{2} \bar{\rho}\right)^{-1} \cdot 2 y\right) \frac{1}{r \bar{\rho}} \frac{d \bar{P}}{d r}, \\
& {[R 5]:=\left(1+\frac{1}{\gamma^{P}} \partial_{z} \Phi^{P}\right)\left(1+\frac{r^{2} v^{2}}{c^{2}}-\frac{2 G m}{c^{2} r(1+y)}\right)\left(1+P / c^{2} \rho\right)^{-1}} \\
& \times \frac{(1+y)^{2}}{r \bar{\rho}} \frac{\partial}{\partial r} \bar{P} \gamma^{P}(3 y+z), \\
& {[R 6]:=\left(1+\frac{r^{2} v^{2}}{c^{2}}-\frac{2 G m}{c^{2} r(1+y)}\right)\left(1+P / c^{2} \rho\right)^{-1}(1+y)^{2} \frac{\bar{P}}{r \bar{\rho}} \cdot[Q 0],} \\
& {[R 7]:=\left(1+\frac{r^{2} v^{2}}{c^{2}}-\frac{2 G m}{c^{2} r(1+y)}\right)\left(1+P / c^{2} \rho\right)^{-1}(1+y)^{2} \frac{1}{r \bar{\rho}} \frac{d \bar{P}}{d r} \cdot[Q 1] .}
\end{aligned}
$$

Then, using (3.1b), we have

$$
[R 1]=[R 3 L]+[R 3 Q]+[R 4 L]+[R 4 Q]+[R 5]+[R 6]+[R 7] .
$$

Let us define $G_{1}$ by

$$
1+G_{1}=\left(1+\frac{1}{\gamma^{P}} \partial_{z} \Phi^{P}\right) \frac{1+\frac{r^{2} v^{2}}{c^{2}}-\frac{2 G m}{c^{2} r(1+y)}}{1-\frac{2 G m}{c^{2} r}} \frac{1+\bar{P} / c^{2} \bar{\rho}}{1+P / c^{2} \rho}(1+y)^{2} .
$$

Then

$$
[R 5]=\left(1+G_{1}\right)\left(1-\frac{2 G m}{c^{2} r}\right)\left(1+\bar{P} / c^{2} \bar{\rho}\right)^{-1} \frac{1}{r \bar{\rho}} \frac{\partial}{\partial r} \bar{P} \gamma^{P}(3 y+z),
$$


and, by definition,

$$
\begin{aligned}
-e^{-2 \bar{F}}\left(1+\bar{P} / c^{2} \bar{\rho}\right)^{-1} \mathcal{L} y= & {[R 3 L]+[R 4 L] } \\
& +\left(1-\frac{2 G m}{c^{2} r}\right)\left(1+\bar{P} / c^{2} \bar{\rho}\right)^{-1} \frac{1}{r \bar{\rho}} \frac{\partial}{\partial r} \bar{P} \gamma^{P}(3 y+z) \\
= & {[R 3 L]+[R 4 L]+\frac{1}{1+G_{1}}[R 5] . }
\end{aligned}
$$

This implies

$$
\begin{aligned}
{[R 1]=} & -\left(1+G_{1}\right) e^{-2 \bar{F}}\left(1+\bar{P} / c^{2} \bar{\rho}\right)^{-1} \mathcal{L} y \\
& -G_{1}([R 3 L]+[R 4 L])+[R 3 Q]+[R 4 Q]+[R 6]+[R 7] .
\end{aligned}
$$

Now, putting

$$
\begin{aligned}
H_{1}:= & e^{F-2 \bar{F}}\left(1+\bar{P} / c^{2} \bar{\rho}\right)^{-1}\left(1+G_{1}\right), \\
H_{2}:= & e^{F} G_{2}, \\
G_{2}:= & \left(1+G_{1}\right)([R 3 L]+[R 4 L])-[R 3]-[R 4] \\
& \quad-[R 6]-[R 7]-[R 2],
\end{aligned}
$$

we can write

$$
e^{F} \times(\text { the right-hand side of }(4.6))=-H_{1} \mathcal{L} y-H_{2} .
$$

The following observation will play a crucial role in the analysis of the equation as in [8].

\section{PROPOSITION 11}

There is an analytic function $\hat{a}$ of $1-x, y, z, v, w, y^{\prime}, y^{\prime \prime}$ such that

$$
\left(\partial_{z} H_{1}\right) \mathcal{L} y+\partial_{z} H_{2}=(1-x) \hat{a}
$$

as $x \rightarrow 1$.

Proof

For the sake of abbreviations, hereafter we will denote

$$
Q_{1} \equiv Q_{0}
$$

if there is an analytic function $\Omega\left(1-x, y, z, v, w, y^{\prime}, y^{\prime \prime}\right)$ such that

$$
Q_{1}=Q_{0}+(1-x) \Omega \text {. }
$$

We are considering

$$
\begin{aligned}
\left(\partial_{z} H_{1}\right) \mathcal{L} y+\partial_{z} H_{2}= & \left(\partial_{z} F \cdot H_{1}+e^{F-2 \bar{F}}\left(1+\bar{P} / c^{2} \rho\right)^{-1} \partial_{z} G_{1}\right) \mathcal{L} y \\
& +\partial_{z} F \cdot H_{2}+e^{F} \partial_{z} G_{2} .
\end{aligned}
$$

First we note that (2.27) and(4.5) imply

$$
\partial_{z} F=\frac{1}{c^{2}} \rho \frac{d u}{d \rho}(1+y+z)^{-1}
$$


and that

$$
\rho \frac{d u}{d \rho}=(\gamma-1) u\left(1+[u]_{1}\right), \quad u=\bar{u}\left(1+\left[(x ; y, z]_{1}\right) \equiv 0 .\right.
$$

(Here $\left[(x ; y, z]_{1}\right.$ stands for an analytic function of $x$ in a neighborhood of $[0,1]$ and $y, z$ in a neighborhood of $(0,0)$ of the form $\sum_{k_{1}+k_{2} \geq 1} a_{k_{1} k_{2}}(x) y^{k_{1}} z^{k_{2}}$.) Therefore, $\partial_{z} F \equiv 0$ and

$$
\left(\partial_{z} H_{1}\right) \mathcal{L} y+\partial_{z} H_{2} \equiv e^{F}[S]
$$

where

$$
\begin{aligned}
{[S]=} & \left(\partial_{z} G_{1}\right) e^{-2 \bar{F}}\left(1+\bar{P} / c^{2} \rho\right)^{-1} \mathcal{L} y+\partial_{z} G_{2} \\
= & -\left(\partial_{z} G_{1}\right)\left(1-\frac{2 G m}{c^{2} r}\right)\left(1+\bar{P} / c^{2} \bar{\rho}\right)^{-1} \frac{1}{r \bar{\rho}} \frac{\partial}{\partial r} \bar{P} \gamma^{P}(3 y+z) \\
& +\left(1+G_{1}\right) \frac{\partial}{\partial z}([R 3 L]+[R 4 L])-\frac{\partial}{\partial z}([R 3]+[R 4]+[R 6]+[R 7]+[R 2]) .
\end{aligned}
$$

But, keeping in mind that $\bar{P} / \bar{\rho} \equiv P / \rho \equiv 0$ and that

$$
\begin{gathered}
\frac{\partial P}{\partial z}=-\rho \frac{d P}{d \rho}(1+y+z)^{-1} \equiv 0, \\
\frac{\partial}{\partial z}\left(\frac{P}{\rho}\right)=\left(-\frac{d P}{d \rho}+\frac{P}{\rho}\right)(1+y+z)^{-1} \equiv 0,
\end{gathered}
$$

we see

$$
\begin{aligned}
& -\left(\partial_{z} G_{1}\right)\left(1-\frac{2 G m}{c^{2} r}\right)\left(1+\bar{P} / c^{2} \bar{\rho}\right)^{-1} \frac{1}{r \bar{\rho}} \frac{\partial}{\partial r} \bar{P} \gamma^{P}(3 y+z) \\
& \equiv-\partial_{z}^{2} \Phi^{P}\left(1+\frac{r^{2} v^{2}}{c^{2}}-\frac{2 G m}{c^{2} r(1+y)}\right)(1+y)^{2} \frac{1}{r \bar{\rho}} \frac{d \bar{P}}{d r}(3 y+z) .
\end{aligned}
$$

On the other hand, it is easy to see

$$
\frac{\partial}{\partial z}[R 3 L] \equiv \frac{\partial}{\partial z}[R 4 L] \equiv \frac{\partial}{\partial z}[R 3] \equiv \frac{\partial}{\partial z}[R 4] \equiv \frac{\partial}{\partial z}[R 6] \equiv \frac{\partial}{\partial z}[R 2] \equiv 0
$$

and

$$
\frac{\partial}{\partial z}[R 7] \equiv-\left(1+\frac{r^{2} v^{2}}{c^{2}}-\frac{2 G m}{c^{2} r(1+y)}\right)(1+y)^{2} \frac{1}{r \bar{\rho}} \frac{d \bar{P}}{d r}(3 y+z) \partial_{z}^{2} \Phi^{P} .
$$

Hence we have $[S] \equiv 0$ so that

$$
\left(\partial_{z} H_{1}\right) \mathcal{L} y+\partial_{z} H_{2} \equiv 0
$$

This was to be shown.

\section{REMARK}

Note that $\partial[R 7] / \partial z \not \equiv 0$. In fact, we have

$$
\frac{1}{r \bar{\rho}} \frac{d \bar{P}}{d r} \rightarrow-\frac{K}{r_{+}} \neq 0
$$


and

$$
\begin{aligned}
\partial_{z}^{2} \Phi^{P} & =-\frac{P}{\rho}\left(\frac{d}{d \rho} \rho \frac{d P}{d \rho}+\frac{d P}{d \rho}\right)(1+y+z)^{-2}\left(1-\gamma^{P}(3 y+z)-\Phi^{P}\right) \\
& \rightarrow-(\gamma+1)(1+y+z)^{-2}\left(1-\gamma(3 y+z)-\Phi^{P}(0, y, z)\right) \neq 0
\end{aligned}
$$

as $x \rightarrow 1$.

Now, putting

$$
J:=e^{F}\left(1+P / c^{2} \rho\right)
$$

we rewrite the system of equations (4.3)-(4.6) as

$$
\begin{aligned}
\frac{\partial y}{\partial t}-J v & =0 \\
\frac{\partial v}{\partial t}+H_{1} \mathcal{L} y+H_{2} & =0
\end{aligned}
$$

Here the unknown functions are $(t, x) \mapsto y, v$.

\section{Framework to apply the Nash-Moser(-Hamilton) theorem}

Having fixed a time-periodic solution $Y_{1}$ of the linearized equation, we put

$$
\begin{aligned}
& y=\varepsilon\left(Y_{1}+Y\right), \\
& z=r \frac{\partial y}{\partial r}=\varepsilon\left(Z_{1}+Z\right) \quad \text { with } Z_{1}=r \frac{\partial Y_{1}}{\partial r}, \\
& v=\varepsilon\left(V_{1}+V\right) \quad \text { with } V_{1}=\frac{1}{J^{o}} \frac{\partial Y_{1}}{\partial t} .
\end{aligned}
$$

Here

$$
J^{o}:=\left.J\right|_{y=z=0}=e^{\bar{F}}\left(1+\bar{P} / c^{2} \bar{\rho}\right),
$$

and $Y, Z=r \partial Y / \partial r$, and $V$ are new unknown functions. The parameter $\varepsilon$ will be taken sufficiently small.

Now the system of equations turns out to be

$$
\begin{gathered}
\frac{\partial Y}{\partial t}-J V-(\Delta J) V_{1}=\left(J-J^{o}\right)^{o} V_{1} \\
\frac{\partial V}{\partial t}+H_{1} \mathcal{L} Y+\left(\Delta H_{1}\right)\left(\mathcal{L} Y_{1}\right)+\frac{1}{\varepsilon} \Delta H_{2}=-\left(H_{1}-\frac{1}{J^{o}}\right)^{o}\left(\mathcal{L} Y_{1}\right)-\frac{1}{\varepsilon} H_{2}^{o}
\end{gathered}
$$

where

$$
\begin{aligned}
\left(J-J^{o}\right)^{o} & :=\left.\left(J-J^{o}\right)\right|_{Y=Z=0}=\left.J\right|_{y=\varepsilon Y_{1}, z=\varepsilon Z_{1}}-J^{o}, \\
\Delta J & :=J-J^{o}-\left(J-J^{o}\right)^{o}=J-\left.J\right|_{y=\varepsilon Y_{1}, z=\varepsilon Z_{1}}, \\
\left(H_{1}-\frac{1}{J^{o}}\right)^{o} & :=\left.\left(H_{1}-\frac{1}{J^{o}}\right)\right|_{Y=Z=V=0} \\
& =\left.H_{1}\right|_{y=\varepsilon Y_{1}, z=\varepsilon Z_{1}, v=\varepsilon V_{1}}-\frac{1}{J^{o}},
\end{aligned}
$$




$$
\begin{aligned}
\Delta H_{1} & :=H_{1}-\frac{1}{J^{o}}-\left(H_{1}-\frac{1}{J^{o}}\right)^{o} \\
& =H_{1}-\left.H_{1}\right|_{y=\varepsilon Y_{1}, z=\varepsilon Z_{1}, v=\varepsilon V_{1},} \\
H_{2}^{o} & :=\left.H_{2}\right|_{y=\varepsilon Y_{1}, z=\varepsilon Z_{1}, v=\varepsilon V_{1}}, \\
\Delta H_{2} & =H_{2}-H_{2}^{o} .
\end{aligned}
$$

Let us introduce the vector-valued unknown function

$$
\vec{w}=\left[\begin{array}{l}
Y \\
V
\end{array}\right] \text {. }
$$

We put

$$
\mathfrak{P}(\vec{w})=\left[\begin{array}{l}
\text { the left-hand side of }(7.5 \mathrm{a}) \\
\text { the left-hand side of }(7.5 \mathrm{~b})
\end{array}\right],
$$

and

$$
\vec{c}=\frac{1}{\varepsilon}\left[\begin{array}{l}
\text { the right-hand side of }(7.5 \mathrm{a}) \\
\text { the right-hand side of }(7.5 \mathrm{~b})
\end{array}\right] .
$$

The equation to be solved now is

$$
\mathfrak{P}(\vec{w})=\varepsilon \vec{c} .
$$

We are going to apply the Nash-Moser(-Hamilton) theorem to find $\vec{w}=$ $\mathfrak{P}^{-1}(\varepsilon \vec{c})$. To do it, we must analyze the Fréchet derivative $D \mathfrak{P}$ of the mapping $\mathfrak{P}$ at a given fixed $\vec{w} \in C^{\infty}\left([0, T]_{t} \times[0,1]_{x}\right)$. By introducing the new variable

$$
\vec{h}=\left[\begin{array}{l}
h \\
k
\end{array}\right]
$$

the Fréchet derivative is defined by

$$
\begin{aligned}
D \mathfrak{P}(\vec{w}) \vec{h} & =\lim _{s \rightarrow 0} \frac{1}{s}(\mathfrak{P}(\vec{w}+s \vec{h})-\mathfrak{P}(\vec{w})) \\
& =\left[\begin{array}{l}
{[D P 1]} \\
{[D P 2]}
\end{array}\right]
\end{aligned}
$$

where

$$
\begin{aligned}
{[D P 1]=} & \frac{\partial}{\partial t} h-J \cdot k \\
& -\left(\left(\partial_{y} J\right) v+\left(\partial_{z} J\right) v r \frac{\partial}{\partial r}\right) h \\
{[D P 2]=} & \frac{\partial}{\partial t} k+H_{1} \cdot \mathcal{L} h \\
& +\left(\left(\partial_{y} H_{1}\right) \mathcal{L} y+\partial_{y} H_{2}+\left(\left(\partial_{z} H_{1}\right) \mathcal{L} y+\partial_{z} H_{2}\right) r \frac{\partial}{\partial r}\right) h \\
& +\left(\left(\partial_{v} H_{1}\right) \mathcal{L} y+\partial_{v} H_{2}+\partial_{w} H_{2} \cdot r \frac{\partial}{\partial r}\right) k .
\end{aligned}
$$

Thanks to Proposition 11, we can claim the following result. 
PROPOSITION 12

We have

$$
\begin{aligned}
\left(\partial_{z} J\right) r \frac{\partial}{\partial r} & =\left[\left(x ; y, D y, D^{2} y, v, D v\right]_{0} \cdot x(1-x) \frac{\partial}{\partial x}\right. \\
\left(\left(\partial_{z} H_{1}\right) \mathcal{L} y+\partial_{z} H_{2}\right) r \frac{\partial}{\partial r} & =\left[\left(x ; y, D y, D^{2} y, v, D v\right]_{0} \cdot x(1-x) \frac{\partial}{\partial x}\right. \\
\partial_{w} H_{2} \cdot r \frac{\partial}{\partial r} & =\left[\left(x ; y, D y, D^{2} y, v, D v\right]_{0} \cdot x(1-x) \frac{\partial}{\partial x}\right.
\end{aligned}
$$

Here $D=\partial / \partial x$.

Proof

Since

$$
r \frac{\partial}{\partial r}=2 x\left(1+[x]_{1}\right) \frac{\partial}{\partial x}
$$

as $x \rightarrow 0(r \rightarrow 0)$, the problem is concentrated to the situation as $x \rightarrow 1\left(r \rightarrow r_{+}\right)$. Now, since

$$
\partial_{z} J=\left(\partial_{z} F\right) J+e^{F} \frac{1}{c^{2}} \frac{\partial}{\partial z}\left(\frac{P}{\rho}\right)=\frac{1}{c^{2}} e^{F} \frac{P}{\rho}(1+y+z)^{-1},
$$

it is clear that $\partial_{z} J \equiv 0(\bmod (1-x))$, that is, (7.14a). From Proposition 11, we have (7.14b). By definition we have

$$
\partial_{w} H_{2}=e^{F} \partial_{w} G_{2}=-e^{F} \partial_{w}[R 2]=-e^{F} \frac{1}{c^{2}}(1+y)^{2} \frac{P}{\bar{\rho}} v \equiv 0,
$$

that is, $(7.14 \mathrm{c})$.

Consequently, we can claim that there are analytic functions $a_{01}, a_{00}, a_{11}, a_{10}$, $a_{21}, a_{20}$ of $x, y, D y, D^{2} y, v, D v$, where $D=\partial / \partial x, y=\varepsilon\left(Y_{1}+Y\right), v=\varepsilon\left(V_{1}+V\right)$, such that the components of $D \mathfrak{P}(\vec{w}) \vec{h}$ can be written as

$$
\begin{aligned}
{[D P 1]=} & \frac{\partial}{\partial t} h-J k+\left(a_{01} x(1-x) D+a_{00}\right) h, \\
{[D P 2]=} & \frac{\partial}{\partial t} k+H_{1} \mathcal{L} h+\left(a_{11} x(1-x) D+a_{10}\right) h \\
& +\left(a_{21} x(1-x) D+a_{20}\right) k .
\end{aligned}
$$

We note that $a_{01}, \ldots, a_{20}=O(\varepsilon)$ provided that $Y, D Y, V, D V=O(1)$. On the other hand we note, by definition, that

$$
J=e^{F}\left(1+P / c^{2} \rho\right)=e^{\bar{F}}\left(1+\bar{P} / c^{2} \bar{\rho}\right)\left(1+\left[(x ; y, D y]_{1}\right)\right.
$$

and

$$
\begin{aligned}
H_{1} & =e^{F-2 \bar{F}}\left(1+\bar{P} / c^{2} \bar{\rho}\right)^{-1}\left(1+G_{1}\right) \\
& =e^{-\bar{F}}\left(1+\bar{P} / c^{2} \bar{\rho}\right)^{-1}\left(1+\left[(x ; y, D y]_{1}+v^{2}\left[(x ; y, D y]_{0}\right) .\right.\right.
\end{aligned}
$$


Hence,

$$
\begin{aligned}
J & =e^{\bar{F}}\left(1+\bar{P} / c^{2} \bar{\rho}\right)(1+O(\varepsilon)), \\
H_{1} & =e^{-\bar{F}}\left(1+\bar{P} / c^{2} \bar{\rho}\right)^{-1}(1+O(\varepsilon))
\end{aligned}
$$

provided that $Y, D Y=O(1)$.

REMARK

We see $J \rightarrow 1, H_{1} \rightarrow 1+[y, z]_{1}$ as $1 / c^{2} \rightarrow 0$, while $\bar{P} / \bar{\rho}, \bar{u}$ are supposed to be bounded. (The equilibrium depends upon the central density $\rho_{c}$ and the speed of light $c$.) But we do not discuss the details of the nonrelativistic limit in this article.

\section{Main conclusion}

Now we are ready to propose the main conclusion of this article.

\section{THEOREM 1}

Given $T>0$, there is a positive number $\varepsilon_{0}(T)$ such that, for $|\varepsilon| \leq \varepsilon_{0}(T)$, there is a solution $\vec{w} \in C^{\infty}([0, T] \times[0,1])$ of (7.10) such that

$$
\sup _{j+k \leq n}\left\|\left(\frac{\partial}{\partial t}\right)^{j}\left(\frac{\partial}{\partial x}\right)^{k} \vec{w}\right\|_{L^{\infty}([0, T] \times[0,1])} \leq C_{n}|\varepsilon|
$$

and, hence, a solution $(y, v) \in C^{\infty}\left([0, T] \times\left[0, r_{+}\right]\right)$of $(4.3)-(4.6)$ of the form

$$
y=\varepsilon Y_{1}+O\left(\varepsilon^{2}\right) .
$$

Note that for this solution the component $R$ of the metric (2.5) behaves like

$$
R=r\left(1+\varepsilon Y_{1}+O\left(\varepsilon^{2}\right)\right),
$$

and the density distribution enjoys

$$
\rho= \begin{cases}C(t)\left(r_{+}-r\right)^{1 /(\gamma-1)}\left(1+O\left(r_{+}-r\right)\right) & 0 \leq r<r_{+} \\ 0 & r_{+} \leq r\end{cases}
$$

Here $C(t)$ is a smooth positive function of $t$.

In other words, the value $R_{+}(t)$ of the Eulerian coordinate $R$ at the surface of the star $r=r_{+}$is approximately oscillating as

$$
R_{+}(t)=r_{+}\left(1+\varepsilon \sin \left(\sqrt{\lambda} t+\Theta_{0}\right) \psi(1)+O\left(\varepsilon^{2}\right)\right) .
$$

A proof can be given by an application of the Nash-Moser(-Hamilton) theorem (see [3, p. 171, Theorem III.1.1.1]) as in [8] and [9]. The discussion is quite parallel. Therefore, omitting the repetitions of the details, we will explain only the points for which some modifications are necessary.

First the mapping $\mathfrak{P}$ is considered on the tame spaces $\overrightarrow{\mathfrak{E}}$ and $\overrightarrow{\mathfrak{E}}_{0}$. Here $\overrightarrow{\mathfrak{E}}=$ $\mathfrak{E} \times \mathfrak{E}$ with $\mathfrak{E}=C^{\infty}([0, T] \times[0,1])$ and $\overrightarrow{\mathfrak{E}}_{0}=\mathfrak{E}_{0} \times \mathfrak{E}_{0}$ with $\mathfrak{E}_{0}=\{\phi \in \mathfrak{E} \mid \phi=$ 
0 at $t=0\}$. Since $\mathfrak{E}$ admits the gradings of norms as in [8], $\overrightarrow{\mathfrak{E}}$ is a tame space as the direct Cartesian product. The domain of $\mathfrak{P}$ is $\overrightarrow{\mathfrak{U}}$, the set of all functions $\vec{w}=(Y, V)^{T} \in \overrightarrow{\mathfrak{E}}_{0}$ such that

$$
|Y|+|D Y|+|V|+|D V|<1 .
$$

We consider $\varepsilon$ such that $|\varepsilon| \leq \varepsilon_{1}$, with $\varepsilon_{1}$ being a fixed sufficiently small positive number. The mapping $\mathfrak{P}$ is a tame mapping from $\overrightarrow{\mathfrak{U}}$ into $\overrightarrow{\mathfrak{E}}$.

Introducing the operator

$$
\Lambda=x(1-x) \frac{d^{2}}{d x^{2}}+\left(\frac{5}{2}(1-x)-\frac{N}{2} x\right) \frac{d}{d x},
$$

just as $[8,(20)]$, we rewrite the second component of $D \mathfrak{P}(\vec{w}) \vec{h}$ as

$$
\begin{aligned}
{[D P 2]=} & \frac{\partial}{\partial t} k-H_{1} \Lambda h \\
& +b_{1} \check{D} h+b_{0} h+a_{21} \check{D} k+a_{20} k,
\end{aligned}
$$

where

$$
\begin{aligned}
\check{D} & =x(1-x) \frac{\partial}{\partial x}, \\
b_{1} & =\frac{H_{1} L_{1}}{x(1-x)}+a_{11}, \\
b_{0} & =H_{1} L_{0}+a_{10} .
\end{aligned}
$$

Then $b_{1}, b_{0}, a_{21}, a_{20}$ are analytic functions of $x, y, D y, D^{2} y, v, D v$. Let us introduce the Hilbert spaces $\mathfrak{X}=\mathfrak{X}^{0}, \mathfrak{X}^{1}, \mathfrak{X}^{2}$, in the same manner as in [8], by

$$
\begin{aligned}
\mathfrak{X} & =L^{2}\left((0,1) ; x^{\frac{3}{2}}(1-x)^{\frac{N}{2}-1} d x\right), \\
\mathfrak{X}^{1} & =\left\{\phi \in \mathfrak{X} \mid \dot{D} \phi:=\sqrt{x(1-x)} \frac{d \phi}{d x} \in \mathfrak{X}\right\}, \\
\mathfrak{X}^{2} & =\left\{\phi \in \mathfrak{X}^{1} \mid-\Lambda \phi \in \mathfrak{X}\right\} .
\end{aligned}
$$

We write the equation

$$
D \mathfrak{P}(\vec{w}) \vec{h}=\vec{g},
$$

where $\vec{g}=\left(g_{1}, g_{2}\right)^{T}$ is a given function in $\overrightarrow{\mathfrak{E}}$, as

$$
\frac{\partial}{\partial t}\left[\begin{array}{l}
h \\
k
\end{array}\right]+\left[\begin{array}{cc}
\mathfrak{a}_{1} & -J \\
\mathcal{A} & \mathfrak{a}_{2}
\end{array}\right]\left[\begin{array}{l}
h \\
k
\end{array}\right]=\left[\begin{array}{l}
g_{1} \\
g_{2}
\end{array}\right],
$$

where

$$
\begin{aligned}
\mathfrak{a}_{1} & =a_{01} \check{D}+a_{00}, \\
\mathfrak{a}_{2} & =a_{21} \check{D}+a_{20}, \\
\mathcal{A} & =-H_{1} \Lambda+b_{1} \check{D}+b_{0} .
\end{aligned}
$$


Then the standard calculation leads us to the equality

$$
\begin{aligned}
\frac{1}{2} \frac{d}{d t} & {\left[\|k\|^{2}+\left(\frac{H_{1}}{J} \dot{D} h \mid \dot{D} h\right)\right] } \\
& +\left(\beta_{1} \dot{D} h \mid \dot{D} h\right)+\left(\beta_{2} \dot{D} h \mid h\right)+\left(\beta_{3} \dot{D} h \mid k\right)+\left(\beta_{4} h \mid k\right)+\left(\beta_{5} k \mid k\right) \\
= & \left(\frac{H_{1}}{J} \dot{D} h \mid \dot{D} g_{1}\right)+\left(k \mid g_{2}\right),
\end{aligned}
$$

where

$$
\begin{aligned}
& \beta_{1}=-\frac{1}{4}(3+(N+3) x+2 \check{D}) \frac{H_{1} a_{01}}{J}-\frac{1}{2} \frac{\partial}{\partial t} \frac{H_{1}}{J}+\frac{H_{1}}{J}\left(\check{D} a_{01}+a_{00}\right), \\
& \beta_{2}=\frac{H_{1}}{J} \dot{D} a_{00}, \\
& \beta_{3}=-\frac{H_{1}}{J} \dot{D} J+\dot{D} H_{1}+\sqrt{x(1-x)}\left(b_{1}+a_{21}\right), \\
& \beta_{4}=b_{0}, \\
& \beta_{5}=a_{20} .
\end{aligned}
$$

Here

$(\phi \mid \psi)=(\phi \mid \psi)_{\mathfrak{X}}=\int_{0}^{1} \phi \bar{\psi} x^{\frac{3}{2}}(1-x)^{\frac{N}{2}-1} d x \quad$ and $\quad\|\phi\|=\|\phi\|_{\mathfrak{X}}=\sqrt{(\phi \mid \phi)_{\mathfrak{X}}}$ and we have used the formula

$$
(\alpha \dot{D} h \mid \dot{D} \check{D} h)=\left(\alpha^{*} \dot{D} h \mid \dot{D} h\right) \quad \text { with } \alpha^{*}=-\frac{1}{4}(3+(N+3) x+2 \check{D}) \alpha,
$$

which holds for $h \in \mathfrak{X}^{2}$ and $\alpha \in C^{\infty}([0,1])$, together with [8, Proposition 8].

Since $\vec{w}$ is confined to $\overrightarrow{\mathfrak{U}}$ and $|\varepsilon|$ is restricted to at most $\varepsilon_{0}$, we can assume

$$
\frac{1}{M_{0}} \leq J \leq M_{0}, \quad \frac{1}{M_{0}} \leq H_{1} \leq M_{0}
$$

with a constant $M_{0}$ independent of $\vec{w}$. Now the energy

$$
\mathcal{E}:=\|k\|^{2}+\left(\frac{H_{1}}{J} \dot{D} h \mid \dot{D} h\right)
$$

enjoys the inequality

$$
\frac{1}{2} \frac{d \mathcal{E}}{d t} \leq M\left(\|\vec{h}\|_{\mathfrak{H}}^{2}+\|\vec{h}\|_{\mathfrak{H}}\|\vec{g}\|_{\mathfrak{H}}\right),
$$

where $\mathfrak{H}=\mathfrak{X}^{1} \times \mathfrak{X}$ and

$$
\left\|(\phi, \psi)^{T}\right\|_{\mathfrak{H}}^{2}=\|\phi\|_{\mathfrak{X}^{1}}^{2}+\|\psi\|_{\mathfrak{X}}^{2}=\|\phi\|^{2}+\|\dot{D} \phi\|^{2}+\|\psi\|^{2},
$$

and

$$
M=\sum_{1 \leq j \leq 5}\left\|\beta_{j}\right\|_{L^{\infty}}+\left(M_{0}\right)^{2}+1
$$

Since

$$
\frac{1}{\left(M_{0}\right)^{2}}\left(\|k\|^{2}+\|\dot{D} h\|^{2}\right) \leq \mathcal{E} \leq\left(M_{0}\right)^{2}\left(\|k\|^{2}+\|\dot{D} h\|^{2}\right),
$$


using the same Gronwall's argument as [8, Proposition 9] and [9, Lemma 3], we see that the initial value problem for (8.4) with the initial condition

$$
h=k=0 \quad \text { at } t=0
$$

admits a unique solution $\vec{h}=(h, k)^{T}$ in $C\left([0, T], \mathfrak{X}^{2} \times \mathfrak{X}^{1}\right)$ for given $\vec{g} \in C([0, T]$, $\left.\mathfrak{X}^{1} \times \mathfrak{X}\right)$, which enjoys the energy estimate

$$
\|\vec{h}\|_{\mathfrak{H}} \leq C \int_{0}^{t}\left\|\vec{g}\left(t^{\prime}\right)\right\|_{\mathfrak{H}} d t^{\prime} .
$$

Therefore, $D \mathfrak{P}(\vec{w})$ admits an inverse, and its tame estimates can be shown in the same manner as in [8]. An outline of this procedure can be found in the Appendix. This completes the proof of the main conclusion.

\section{Cauchy problems}

As a supplement let us consider the Cauchy problem associated with equations (6.10a)-(6.10b), that is, $(\mathrm{CP})$ :

$$
\begin{aligned}
\frac{\partial y}{\partial t}-J v & =0, \quad \frac{\partial v}{\partial t}+H_{1} \mathcal{L} y+H_{2}=0, \quad t \geq 0,0 \leq x \leq 1, \\
\left.y\right|_{t=0} & =\psi_{0}(x),\left.\quad v\right|_{t=0}=\psi_{1}(x) .
\end{aligned}
$$

Here $\psi_{0}$ and $\psi_{1}$ are functions given in $C^{\infty}([0,1])$.

Let us recall that

$$
J=e^{F}\left(1+P / c^{2} \rho\right)=J(x, y, z)
$$

is an analytic function of $x$ (in a neighborhood of $[0,1]$ ), $y$ (small), and $z=$ $r \frac{\partial y}{\partial r}=x[(x)] \frac{\partial y}{\partial x}$ (small), where $[(x)]$ stands for an analytic function of $x$ in a neighborhood of $[0,1]$. Recall that $H_{1}$ and $H_{2}$ are analytic functions of $x, y, z$, $v$, and $w=r \frac{\partial v}{\partial r}$ (quadratic in $v / c, w / c$ ), and recall that the linear operator $\mathcal{L}$ has the form

$$
\begin{aligned}
\mathcal{L} y= & -x(1-x) \frac{d^{2} y}{d x^{2}}-\left(\frac{5}{2}(1-x)-\frac{N}{2} x\right) \frac{d y}{d x} \\
& +l_{1}(x) x(1-x) \frac{d y}{d x}+L_{0}(x) y,
\end{aligned}
$$

where $l_{1}$ and $L_{0}$ are analytic functions of $x$ in a neighborhood of $[0,1]$.

We claim the following result.

\section{THEOREM 2}

For any given $T>0$ there exists a sufficiently small positive number $\delta$ such that if $\psi_{0}, \psi_{1} \in C^{\infty}([0,1])$ satisfy

$$
\max _{k \leq \mathfrak{K}}\left\{\left\|\left(\frac{d}{d x}\right)^{k} \psi_{0}\right\|_{L^{\infty}},\left\|\left(\frac{d}{d x}\right)^{k} \psi_{1}\right\|_{L^{\infty}}\right\} \leq \delta,
$$

then there exists a unique solution $(y, v)$ of $(C P)$ in $C^{\infty}([0, T] \times[0,1])$. Here $\mathfrak{K}$ is a sufficiently large number depending only upon $\gamma$. 
Proof

The proof can be done in almost the same manner as that of [8, Theorem 2]. Let us take the functions

$$
y_{1}^{*}=\psi_{0}(x)+t J^{o}(x) \psi_{1}(x), \quad v_{1}^{*}=\psi_{1}(x),
$$

which satisfy the initial conditions, where

$$
J^{o}(x)=J(x, 0,0)
$$

as in (7.4). Then we seek a solution $(y, v)$ of the form

$$
y=y_{1}^{*}+Y, \quad v=v_{1}^{*}+V .
$$

The initial condition for $\vec{w}:=(Y, V)^{T}$ is

$$
\left.\vec{w}\right|_{t=0}=(0,0)^{T}
$$

and the equations to be satisfied by $\vec{w}=(Y, V)^{T}$ are

$$
\begin{aligned}
\frac{\partial Y}{\partial t}-J V-(\Delta J) v_{1}^{*} & =c_{1}, \\
\frac{\partial V}{\partial t}+H_{1} \mathcal{L} Y+\left(\Delta H_{1}\right) \mathcal{L} y_{1}^{*}+\Delta H_{2} & =c_{2},
\end{aligned}
$$

where

(9.7d) $\quad H_{1}=H_{1}\left(x, y_{1}^{*}+Y, z_{1}^{*}+Z, v_{1}^{*}+V, w_{1}^{*}+W\right), \quad$ with $W=r \frac{\partial V}{\partial r}$,

(9.7e) $\Delta H_{1}=H_{1}\left(x, y_{1}^{*}+Y, z_{1}^{*}+Z, v_{1}^{*}+V, w_{1}^{*}+W\right)-H_{1}\left(x, y_{1}^{*}, z_{1}^{*}, v_{1}^{*}, w_{1}^{*}\right)$,

(9.7f) $\Delta H_{2}=H_{2}\left(x, y_{1}^{*}+Y, z_{1}^{*}+Z, v_{1}^{*}+V, w_{1}^{*}+W\right)-H_{2}\left(x, y_{1}^{*}, z_{1}^{*}, v_{1}^{*}, w_{1}^{*}\right)$,

$$
c_{2}=-H_{1}\left(x, y_{1}^{*}, z_{1}^{*}, v_{1}^{*}, w_{1}^{*}\right) \mathcal{L} y_{1}^{*}-H_{2}\left(x, y_{1}^{*}, z_{1}^{*}, v_{1}^{*}, w_{1}^{*}\right) .
$$

The problem can be written as

$$
\mathfrak{P}(\vec{w})=\vec{c},
$$

where

$$
\begin{aligned}
\mathfrak{P}(\vec{w}) & =\left[\begin{array}{l}
\text { the left-hand side of }(9.6 \mathrm{a}) \\
\text { the left-hand side of }(9.6 \mathrm{~b})
\end{array}\right], \\
\vec{c} & =\left[\begin{array}{l}
c_{1} \\
c_{2}
\end{array}\right] .
\end{aligned}
$$

Then the Nash-Moser(-Hamilton) theorem can be applied in the same manner as the proof of Theorem 1 , since the Fréchet derivative of $\mathfrak{P}$ has the same form as (7.12)-(7.13). This completes the proof. 


\section{REMARK}

The initial data read

$$
\begin{aligned}
\left.R\right|_{t=0} & =r\left(1+\psi_{0}(x(r))\right), \\
\left.\frac{\partial R}{\partial t}\right|_{t=0} & =\frac{1}{c} \sqrt{1-\frac{2 G m_{+}}{c^{2} r_{+}}} \exp \left[-\frac{1}{c^{2}} u\left(\rho^{0}\right)\right] r \psi_{1}(x(r)),
\end{aligned}
$$

where

$$
\rho^{0}=\bar{\rho}(r)\left(1+\psi_{0}\right)^{-2}\left(1+\psi_{0}+r \frac{d \psi_{0}}{d r}\right)^{-1}
$$

\section{SUPPLEMENTARY REMARK 4}

Let us consider moving solutions constructed as in Sections 8 and 9, which are defined on $0 \leq t \leq T, 0 \leq r \leq r_{+}$. We should discuss how to extend the metric onto the exterior vacuum region $r>r_{+}$. We owe the idea to [10].

If a spherically symmetric extension to the vacuum region is possible, then Birkhoff's theorem says that it should be the Schwartzschild metric

$$
d s^{2}=\left(1-\frac{2 G m_{+}}{c^{2} R^{\sharp}}\right) c^{2}\left(d t^{\sharp}\right)^{2}-\left(1-\frac{2 G m_{+}}{c^{2} R^{\sharp}}\right)^{-1}\left(d R^{\sharp}\right)^{2}-\left(R^{\sharp}\right)^{2}\left(d \theta^{2}+\sin ^{2} \theta d \phi^{2}\right) .
$$

Here $t^{\sharp}=t^{\sharp}(t, r)$ and $R^{\sharp}=R^{\sharp}(t, r)$ are smooth functions of $0 \leq t \leq T$ and $r_{+} \leq$ $r<\infty$. We have that there are $t^{\sharp}(t, r), R^{\sharp}(t, r)$ such that the components of the metric are of class $C^{1}([0, T] \times[0,+\infty))$.

Let us verify this. We are considering the patched metric

$$
d s^{2}=g_{00} c^{2} d t^{2}+2 g_{01} c d t d r+g_{11} d r^{2}+g_{22}\left(d \theta^{2}+\sin ^{2} \theta d \phi^{2}\right),
$$

where

$$
\begin{aligned}
& g_{00}= \begin{cases}\kappa e^{-2 u / c^{2}} & 0 \leq r \leq r_{+}, \\
K^{\sharp}\left(\frac{\partial t^{\sharp}}{\partial t}\right)^{2}-\frac{1}{c^{2}}\left(K^{\sharp}\right)^{-1}\left(\frac{\partial R^{\sharp}}{\partial t}\right)^{2} & r_{+}<r,\end{cases} \\
& g_{01}= \begin{cases}0 & 0 \leq r \leq r_{+}, \\
c K^{\sharp} \frac{\partial t^{\sharp}}{\partial t} \frac{\partial t^{\sharp}}{\partial r}-\frac{1}{c}\left(K^{\sharp}\right)^{-1} \frac{\partial R^{\sharp}}{\partial t} \frac{\partial R^{\sharp}}{\partial r} & r_{+}<r,\end{cases} \\
& g_{11}= \begin{cases}-\left(1+\frac{V^{2}}{c^{2}}-\frac{2 G m}{c^{2}}\right)^{-1}\left(\frac{\partial R}{\partial r}\right)^{2} & 0 \leq r \leq r_{+}, \\
c^{2} K^{\sharp}\left(\frac{\partial t^{\sharp}}{\partial r}\right)^{2}-\left(K^{\sharp}\right)^{-1}\left(\frac{\partial R^{\sharp}}{\partial r}\right)^{2} & r_{+}<r,\end{cases} \\
& g_{22}= \begin{cases}-R^{2} & 0 \leq t \leq r_{+}, \\
-\left(R^{\sharp}\right)^{2} & r_{+}<r .\end{cases}
\end{aligned}
$$

Here

$$
K^{\sharp}=1-\frac{2 G m_{+}}{c^{2} R^{\sharp}} .
$$

Let us assume $R=R^{\sharp}$ and $\partial_{r} R=\partial_{r} R^{\sharp}$ at $r=r_{+}$so that $g_{22}$ will be of class $C^{1}$. 
First, in order for $g_{00}$ to be continuous across $r=r_{+}$, we require

$$
\frac{\partial t^{\sharp}}{\partial t}=\sqrt{\kappa}\left(K^{\sharp}\right)^{-1}\left(1+\frac{V^{2}}{c^{2}}-\frac{2 G m_{+}}{c^{2} R}\right)^{1 / 2},
$$

on $r=r_{+}$, where $V=V\left(t, r_{+}-0\right)\left(=\frac{1}{\sqrt{\kappa}} \frac{\partial R}{\partial t}\right)$. In order for $g_{01}$ to be continuous, we require

$$
\frac{\partial t^{\sharp}}{\partial r}=\frac{1}{c^{2}}\left(K^{\sharp}\right)^{-1}\left(1+\frac{V^{2}}{c^{2}}-\frac{2 G m_{+}}{c^{2} R}\right)^{-1 / 2} V \frac{\partial R}{\partial r}
$$

on $r=r_{+}$. It can be shown that (9.12) is sufficient in order for $g_{11}$ to be continuous across $r=r_{+}$. In summary, the $g_{\mu \nu}$ 's are continuous if (9.11) and (9.12) hold. Note that, since

$$
K^{\sharp} \doteqdot \kappa, \quad 1+\frac{V^{2}}{c^{2}}-\frac{G m_{+}}{c^{2} R} \doteqdot \kappa, \quad \frac{\partial R}{\partial r} \doteqdot 1,
$$

the right-hand side of $(9.12) \doteqdot V / c^{2}$ so that $\partial t^{\sharp} / \partial r \neq 0$ and $t^{\sharp}$ should actually depend upon $r$ if $V \neq 0$, that is, if the solution is actually moving.

By a tedious calculation we can show that the differentiation of (9.11) with respect to $t$ gives the continuity of $\partial_{r} g_{00}$. On the other hand the continuity of $\partial_{r} g_{01}$ reads as a condition of the form

$$
K^{\sharp} \frac{\partial t^{\sharp}}{\partial t} \frac{\partial^{2} t^{\sharp}}{\partial r^{2}}-\frac{1}{c^{2}}\left(K^{\sharp}\right)^{-1} \frac{\partial R}{\partial t} \frac{\partial^{2} R^{\sharp}}{\partial r^{2}}=b_{1}
$$

on $r=r_{+}$, where $b_{1}$ is a function of the values of $\partial_{t} t^{\sharp}, \partial_{r} t^{\sharp}, \partial_{t} \partial_{r} t^{\sharp}, R, \partial_{r} R, \partial_{t} \partial_{r} R$ on $r=r_{+}$. The continuity of $\partial_{r} g_{11}$ reads as a condition of the form

$$
c^{2} K^{\sharp} \frac{\partial t^{\sharp}}{\partial r} \frac{\partial^{2} t^{\sharp}}{\partial r^{2}}-\left(K^{\sharp}\right)^{-1} \frac{\partial R}{\partial r} \frac{\partial^{2} R^{\sharp}}{\partial r^{2}}=b_{2},
$$

on $r=r_{+}$, where $b_{2}$ is a function of the same kind as $b_{1}$. If we consider (9.13)(9.14) as a system of simultaneous linear equations for the unknown $\partial^{2} t^{\sharp} / \partial r^{2}$, $\partial^{2} R^{\sharp} / \partial r^{2}$, then the determinant of the coefficient matrix is

$$
-\sqrt{\kappa}\left(1+\frac{V^{2}}{c^{2}}-\frac{2 G m_{+}}{c^{2} R}\right)^{-1 / 2} \frac{\partial R}{\partial r}
$$

which is near to -1 , since

$$
1+\frac{V^{2}}{c^{2}}-\frac{2 G m_{+}}{c^{2} R} \doteqdot \kappa, \quad \frac{\partial R}{\partial r} \doteqdot 1
$$

Since $b_{1}, b_{2}$ are known by (9.11)-(9.12), the values $\partial^{2} t^{\sharp} / \partial r^{2}, \partial^{2} R^{\sharp} / \partial r^{2}$ along $r=r_{+}+0$ are uniquely determined. Then all $g_{\mu \nu}$ 's are of class $C^{1}$.

However, we note that this $\partial^{2} R^{\sharp} / \partial r^{2}$ generally does not coincide with $\partial^{2} R / \partial r^{2}$ on $r=r_{+}$, which is necessary for $g_{22}$ to be twice continuously differentiable. In fact by a tedious calculation we get

$$
\left.\frac{\partial^{2} R^{\sharp}}{\partial r^{2}}\right|_{r=r_{+}+0}=\mathcal{A}\left(\frac{\partial R}{\partial r}\right)^{2}+\left.\frac{\partial^{2} R}{\partial r^{2}}\right|_{r=r_{+}-0},
$$

where

$$
\mathcal{A}=-\frac{V^{2}}{c^{2}}\left(\frac{G m_{+}}{c^{2} R^{2}}+\frac{1}{\sqrt{\kappa}} \frac{1}{c^{2}} \frac{\partial V}{\partial t}\right)\left(1+\frac{V^{2}}{c^{2}}-\frac{2 G m_{+}}{c^{2} R}\right)^{-2}
$$


evaluated at $r=r_{+}-0$. Since

$$
\frac{G m_{+}}{c^{2} R^{2}}\left(1+\frac{V^{2}}{c^{2}}-\frac{2 G m_{+}}{c^{2} R}\right)^{-2} \doteqdot \frac{G m_{+}}{c^{2} r_{+}^{2} \kappa^{2}} \neq 0,
$$

we see that $\partial^{2} R^{\sharp} / \partial r^{2} \equiv \partial^{2} R / \partial r^{2}$ if and only if $V \equiv 0$ at $r=r_{+}-0$, which is the case if the solution under consideration is an equilibrium.

We have determined the functions

$$
\begin{aligned}
f_{0}(t) & :=R\left(t, r_{+}\right), \quad f_{1}(t):=\partial_{r} R\left(t, r_{+}\right), \\
f_{2}(t) & :=\frac{\partial^{2} R^{\sharp}}{\partial r^{2}} \quad \text { at } r=r_{+}+0, \\
H(t) & :=\frac{\partial t^{\sharp}}{\partial t} \quad \text { at } r=R_{+}+0, \quad h_{0}(t):=\int_{0}^{t} H\left(t^{\prime}\right) d t^{\prime}, \\
h_{1}(t) & :=\frac{\partial t^{\sharp}}{\partial r} \quad \text { at } r=r_{+}+0, \quad h_{2}(t):=\frac{\partial^{2} t^{\sharp}}{\partial r^{2}} \quad \text { at } r=r_{+}+0
\end{aligned}
$$

for $0 \leq t \leq T$. Using these functions we define $t^{\sharp}(t, r), R^{\sharp}(t, r)$ for $0 \leq t \leq T, r_{+} \leq$ $r<+\infty$ as follows:

$$
\begin{aligned}
R^{\sharp}(t, r) & =f_{0}(t)+f_{1}(t)\left(r-r_{+}\right)+\frac{1}{2} f_{2}(t)\left(r-r_{+}\right)^{2} \chi\left(r-r_{+}\right), \\
t^{\sharp}(t, r) & =h_{0}(t)+\left(h_{1}(t)\left(r-r_{+}\right)+\frac{1}{2} h_{2}(t)\left(r-r_{+}\right)^{2}\right) \chi\left(\delta\left(r-r_{+}\right)\right) .
\end{aligned}
$$

Here $\chi$ is a smooth cutoff function in $C^{\infty}[0,+\infty)$ such that $0 \leq \chi(s) \leq 1, \chi(s)=1$ for $0 \leq s \leq 1$, and $\chi(s)=0$ for $2 \leq s<+\infty$ and $\delta$ is a sufficiently small positive number. Since $f_{0}(t) \doteqdot r_{+}, f_{1}(t) \doteqdot 1, f_{2}(t) \doteqdot 0, H(t) \doteqdot 1$, we see that $\partial R^{\sharp} / \partial r \doteqdot 1$ and $\partial t^{\sharp} / \partial t \doteqdot 1$ uniformly. Then the coefficients of the metric $g_{00}, g_{01}, g_{11}$, and $g_{22}$ are of class $C^{1}([0, T] \times[0,+\infty))$ and their second-order derivatives may have discontinuity of at most the first kind along the segment $r=r_{+}$, and they satisfy the Einstein equations in the usual sense on $r \neq r_{+}$. So, we can say that this metric is a weak solution of the Einstein equations on $[0, T] \times \mathbb{R}^{3}$ in the following sense. The Einstein equations can be written as

$$
R_{\mu \nu}=\frac{8 \pi G}{c^{4}}\left(T_{\mu \nu}-\frac{1}{2} g_{\mu \nu} T\right)
$$

and

$$
\begin{aligned}
T= & T^{\alpha \beta} T_{\alpha \beta}, \\
R_{\mu \nu}= & \frac{1}{2} g^{\alpha \beta}\left(-\partial_{\alpha} \partial_{\beta} g_{\mu \nu}-\partial_{\mu} \partial_{\nu} g_{\alpha \beta}+\partial_{\beta} \partial_{\nu} g_{\mu \alpha}+\partial_{\mu} \partial_{\alpha} g_{\beta \nu}\right)+F_{\mu \nu}, \\
F_{\mu \nu}= & \frac{1}{2} \partial_{\alpha} g^{\alpha \beta}\left(\partial_{\nu} g_{\beta \mu}+\partial_{\mu} g_{\beta \nu}-\partial_{\beta} g_{\mu \nu}\right) \\
& -\frac{1}{2} \partial_{\nu} g^{\alpha \beta}\left(\partial_{\alpha} g_{\beta \mu}+\partial_{\mu} g_{\beta \alpha}-\partial_{\beta} g_{\mu \alpha}\right) .
\end{aligned}
$$


Therefore, for $\left(T_{\mu \nu}\right)_{\mu \nu}, T \in L_{\mathrm{loc}}^{2}$ given, $d s^{2}=g_{\mu \nu} d x^{\mu} d x^{\nu}$ is said to be a weak solution if $g_{\mu \nu}, g^{\alpha \beta} \in H_{\text {loc }}^{1}$ and for any test function $\left(\phi^{\mu \nu}\right)_{\mu \nu}$ there holds

$$
\begin{aligned}
& \frac{1}{2} \int\left(\partial_{\beta} g_{\mu \nu}\right) \partial_{\alpha}\left(g^{\alpha \beta} \phi^{\mu \nu}\right)+\left(\partial_{\nu} g_{\alpha \beta}\right) \partial_{\mu}\left(g^{\alpha \beta} \phi^{\mu \nu}\right) \\
& \quad-\left(\partial_{\nu} g_{\mu \alpha}\right) \partial_{\beta}\left(g^{\alpha \beta} \phi^{\mu \nu}\right)-\left(\partial_{\alpha} g_{\beta \nu}\right) \partial_{\mu}\left(g^{\alpha \beta} \phi^{\mu \nu}\right)+\int F_{\mu \nu} \phi^{\mu \nu} \\
& =\frac{8 \pi G}{c^{4}} \int\left(T_{\mu \nu}-\frac{1}{2} g_{\mu \nu} T\right) \phi^{\mu \nu}
\end{aligned}
$$

\section{Appendix}

Let us give an outline of the tame estimate of the mapping $(\vec{w}, \vec{g}) \mapsto \vec{h}$ when $D \mathfrak{P}(\vec{w}) \vec{h}=\vec{g}$. The equation (8.4) is split as [8] using a cutoff function $\omega \in C^{\infty}$ such that $\omega(x)=1$ for $x \leq 1 / 3,0<\omega(x)<1$ for $1 / 3<x<2 / 3$, and $\omega(x)=0$ for $2 / 3 \leq x$. Put

$$
\vec{h}^{[0]}(x)=\omega(x) \vec{h}(x), \quad \vec{h}^{[1]}(x)=(1-\omega(x)) \vec{h}(x) .
$$

The equations turn out to be

$$
\begin{aligned}
\frac{\partial}{\partial t}\left[\begin{array}{l}
h^{[\mu]} \\
k^{[\mu]}
\end{array}\right]+\left[\begin{array}{cc}
\mathfrak{a}_{1}^{[\mu]} & -J \\
\mathcal{A}^{[\mu]} & \mathfrak{a}_{2}^{[\mu]}
\end{array}\right]\left[\begin{array}{c}
h^{[\mu]} \\
k^{[\mu]}
\end{array}\right] \\
=\left[\begin{array}{c}
g_{1}^{[\mu]} \\
g_{2}^{[\mu]}
\end{array}\right]+(-1)^{\mu}\left[\begin{array}{cc}
c_{11} & 0 \\
\mathfrak{c}_{21} & c_{22}
\end{array}\right]\left[\begin{array}{l}
h^{[1-\mu]} \\
k^{[1-\mu]}
\end{array}\right],
\end{aligned}
$$

where $\mu=0,1$ and

$$
\begin{aligned}
\mathfrak{a}_{1}^{[\mu]} & =a_{01} \check{D}+a_{00}-(-1)^{\mu} a_{01} \check{D} \omega, \\
\mathfrak{a}_{2}^{[\mu]} & =a_{21} \check{D}+a_{20}-(-1)^{\mu} a_{21} \check{D} \omega, \\
\mathcal{A}^{[\mu]} & =-H_{1} \Lambda+\left(b_{1}+(-1)^{\mu} 2 H_{1}(D \omega)\right) \check{D}+b_{0}+(-1)^{\mu}\left(H_{1} \Lambda-b_{1} \check{D}\right) \omega, \\
c_{11} & =a_{01} \check{D} \omega \\
\mathfrak{c}_{21} & =-2 H_{1}(D \omega) \check{D}+b_{1}(\check{D} \omega)-H_{1}(\Lambda \omega), \\
c_{22} & =a_{21} \check{D} \omega .
\end{aligned}
$$

Therefore, the problem is reduced to the tame estimate of an equation of the form

$$
\begin{aligned}
\frac{\partial \vec{h}}{\partial t}+\mathfrak{A} \vec{h} & =\vec{g} \\
\mathfrak{A} & =\left[\begin{array}{cc}
\mathfrak{a}_{1} & J \\
\mathcal{A} & \mathfrak{a}_{2}
\end{array}\right]=\left[\begin{array}{cc}
a_{01} \check{D}+a_{00} & J \\
-b_{2} \Delta+b_{1} \check{D}+b_{0} & a_{21} \check{D}+a_{20}
\end{array}\right],
\end{aligned}
$$

under the boundary condition $\left.h\right|_{x=1}=0$, where

$$
\triangle=x \frac{d^{2}}{d x^{2}}+\frac{N}{2} \frac{d}{d x}, \quad \check{D}=x \frac{d}{d x}
$$

with $N$ standing for either $2 \gamma /(\gamma-1)$ or 5 . 
As in [8], we use the notations

$$
\begin{aligned}
\vec{a} & =\left(a_{i}\right)_{i=0}^{7}=\left(b_{0}, b_{1}, b_{2}, a_{01}, a_{00}, a_{21}, a_{20}, J\right), \\
|\vec{a}|_{n}^{\langle T\rangle} & =\sup _{0 \leq t \leq T}|\vec{a}|_{n}, \\
|\vec{a}|_{n} & =\max _{j+k \leq n, 0 \leq i \leq 7}\left\|\partial_{t}^{j} \dot{D}^{k} a_{i}\right\|_{L^{\infty}}, \\
\|\vec{h}\|_{n}^{\langle T\rangle} & =\left(\sum_{j+k \leq n} \int_{0}^{T}\left\|\partial_{t}^{j} \vec{h}\right\|_{k}^{2} d t\right)^{1 / 2}, \\
\|\vec{h}\|_{k} & =\left(\sum_{0 \leq \ell \leq k}\langle h\rangle_{\ell+1}^{2}+\langle k\rangle_{\ell}^{2}\right)^{1 / 2} .
\end{aligned}
$$

Here $\langle\phi\rangle_{\ell}$ means the same as it does in [9].

Then the elliptic a priori estimate [9, Proposition 8] should read

$$
\|\vec{h}\|_{n+1} \leq C\left(\|\mathfrak{A} \vec{h}\|_{n}+\left(1+|\vec{a}|_{n+4}\right)\|\vec{h}\|_{0}\right) .
$$

This can be verified if we keep in mind that

$$
\begin{gathered}
\left\|\mathfrak{a}_{1} h\right\|_{1} \leq C\left(|\varepsilon|\|h\|_{2}+\|h\|_{1}\right), \\
\left\|\mathfrak{a}_{2} k\right\|_{0} \leq C\left(|\varepsilon|\|k\|_{1}+\|k\|_{0}\right),
\end{gathered}
$$

which come from

$$
\begin{aligned}
& a_{01}=\frac{1}{c^{2}} e^{F} \frac{P}{\rho}(1+y+z)^{-1} \varepsilon\left(V_{1}+V\right) \frac{r}{x(1-x)} \frac{d x}{d r}, \\
& a_{21}=-\frac{1}{c^{2}} e^{F} \frac{P}{\bar{\rho}}(1+y)^{2} \varepsilon\left(V_{1}+V\right) \frac{r}{x(1-x)} \frac{d x}{d r} .
\end{aligned}
$$

In fact, estimates of the commutators

$$
\begin{gathered}
\|[\triangle, \mathcal{A}] \phi\|_{n} \leq C\left(|\vec{a}|_{2}\|\phi\|_{n+3}+|\vec{a}|_{n+5}\|\phi\|_{0}\right), \\
\|[\triangle, \mathfrak{a}], \phi\|_{n} \leq C\left(|\vec{a}|_{3}\|\phi\|_{n+2}+|\vec{a}|_{n+5}\|\phi\|_{0}\right), \\
\|[\triangle, J] \phi\|_{n} \leq C\left(|\vec{a}|_{4}\|\phi\|_{n+1}+|\vec{a}|_{n+5}\|\phi\|_{0}\right)
\end{gathered}
$$

can be derived as in [9] and used to prove the elliptic a priori estimate by induction on $n$.

On the other hand, the energy estimate should read

$$
\|\vec{H}\| \leq C\left(\left\|\left.\vec{H}\right|_{t=0}\right\|+\int_{0}^{T}\left\|\vec{G}\left(t^{\prime}\right)\right\| d t^{\prime}\right),
$$

where

$$
\|\vec{H}\|=\left(\|H\|^{2}+\|\dot{D} H\|^{2}+\|K\|^{2}\right)^{1 / 2} \quad \text { with }\|\cdot\|=\|\cdot\|_{L^{2}\left(x^{\frac{N}{2}-1} d x\right)},
$$

for any solution $\vec{H}=(H, K)^{T}$ of

$$
\frac{\partial \vec{H}}{\partial t}+\mathfrak{A} \vec{H}=\vec{G},\left.\quad H\right|_{x=1}=0,
$$


which may not vanish at $t=0$, so that

$$
\left\|\partial_{t}^{n} \vec{h}\right\| \leq C\left(\left\|\left.\partial_{t}^{n} \vec{h}\right|_{t=0}\right\|+\int_{0}^{t}\left\|\partial_{t}^{n} \vec{g}\right\|+\int_{0}^{t}\left\|\left[\partial_{t}^{n}, \mathfrak{A}\right] \vec{h}\right\|\right) .
$$

Moreover, we have an estimate

$$
\left\|\left.\partial_{t}^{n+1} \vec{h}\right|_{t=0}\right\| \leq C\left(1+W_{n}(\vec{g})+|\vec{a}|_{n+3}^{\langle 0\rangle}\right),
$$

where

$$
W_{n}(\vec{g})=\sum_{j+k \leq n}\left\|\left.\partial_{t}^{j} \vec{g}\right|_{t=0}\right\|_{k},
$$

provided that $|\vec{a}|_{4}$ and $W_{0}(\vec{g})$ are bounded. In order to verify this, it is sufficient to show

$$
\left\|\left.\partial_{t}^{n+1} \vec{h}\right|_{t=0}\right\|_{k} \leq C\left(W_{n+k}(\vec{g})+|\vec{a}|_{n+k+3}^{\langle 0\rangle} W_{0}(\vec{g})+|\vec{a}|_{k+4}^{\langle 0\rangle} W_{n-1}(\vec{g})\right)
$$

inductively on $n$ using

$$
\|\mathfrak{A} \vec{h}\|_{n} \leq C\left(\|\vec{h}\|_{n+1}+|\vec{a}|_{n+4}\|\vec{h}\|_{0}\right) .
$$

Then the same discussion using the auxiliary quantity

$$
Z_{n}(\vec{h})=\sum_{j+k=n}\left\|\partial_{t}^{j} \vec{h}\right\|_{k}
$$

as in [9] leads us to the estimate

$$
\|\vec{h}\|_{n+1}^{\langle t\rangle} \leq C\left(1+\int_{0}^{t}\|\vec{g}\|_{n+1}^{\left\langle t^{\prime}\right\rangle} d t^{\prime}+W_{n}(\vec{g})+\|\vec{g}\|_{n}^{\langle T\rangle}+|\vec{a}|_{n+3}^{\langle T\rangle}\right)
$$

for $0 \leq t \leq T$.

This estimate for the split problem is sufficient to get the tame estimate for the original $\vec{h}=\vec{h}^{[0]}+\vec{h}^{[1]}$ as in [8]. We omit the repetition of the discussion.

Acknowledgments. A part of this work was done when the author stayed at Université de Strasbourg for "Rencontre entre mathématiciens et physiciens théoriciens: Riemann, Einstein et la geométrie." The author expresses his thanks to the organizers Athanase Papadopoulos (Strasbourg) and Sumio Yamada (Tokyo) for their hospitality. The author expresses his sincere thanks to the anonymous referees for a careful reading of the original manuscript and many kind suggestions for improving the exposition.

\section{References}

[1] G. Birkhoff and G.-C. Rota, Ordinary Differential Equations, 3rd ed., Wiley, New York, 1978. MR 0507190.

[2] U. Brauer and L. Karp, Local existence of solutions of self gravitating relativistic perfect fluids, Comm. Math. Phys. 325 (2014), 105-141.

MR 3182488. DOI 10.1007/s00220-013-1854-3. 
[3] R. S. Hamilton, The inverse function theorem of Nash and Moser, Bull. Amer. Math. Soc. (N.S.) 7 (1982), 65-222. MR 0656198.

DOI 10.1090/S0273-0979-1982-15004-2.

[4] M. Hukuhara, T. Kimura, and T. Matuda, Equations différentielles ordinaires du premier ordre dans le champ complexe, Publ. Math. Soc. Japan, Tokyo, 1961. MR 0124549.

[5] L. D. Landau and E. M. Lifshitz, Course of Theoretical Physics, Vol. 2, 4th ed., Pergamon Press, Oxford, 1975. MR 0475345.

[6] T. Makino, "On a local existence theorem for the evolution equation of gaseous stars" in Patterns and Waves, Stud. Math. Appl. 18, North-Holland, Amsterdam, 1986, 459-479. MR 0882389. DOI 10.1016/S0168-2024(08)70142-5.

[7]_ On spherically symmetric stellar models in general relativity, Kyoto J. Math. 38 (1998), 55-69. MR 1628067.

[8] - On spherically symmetric motions of a gaseous star governed by the Euler-Poisson equations, Osaka J. Math. 52 (2015), 545-580. MR 3326626.

[9]_ On spherically symmetric motions of the atmosphere surrounding a planet governed by the compressible Euler equations, Funkcial. Ekvac. $\mathbf{5 8}$ (2015), 43-85. MR 3379135.

[10] C. W. Misner and D. H. Sharp, Relativistic equations for adiabatic, spherically symmetric gravitational collapse, Phys. Rev. (2) 136 (1964), B571-B576. MR 0177783.

[11] C. W. Misner, K. S. Thorne, and J. A. Wheeler, Gravitation, Freeman, San Francisco, Calif., 1970. MR 0418833.

[12] J. P. Oppenheimer and G. M. Volkoff, On massive neutron cores, Phys. Rev. 55 (1939), 374-381. DOI 10.1103/PhysRev.55.374.

[13] M. Reed and B. Simon, Methods of Modern Mathematical Physics, II: Fourier Analysis, Self-Adjointness, Academic Press, New York, 1975. MR 0493420.

[14] A. D. Rendall and B. G. Schmidt, Existence and properties of spherically symmetric static fluid bodies with a given equation of state, Class. Quantum Gravity 8 (1991), 985-1000. MR 1104769.

[15] Ya. B. Zeldovich and I. D. Novikov, Relativistic Astrophysics, 1: Stars and Relativity, Univ. Chicago Press, Chicago, 1971.

Department of Applied Mathematics, Yamaguchi University, Ube, Japan; makino@yamaguchi-u.ac.jp 